\title{
Theory, modeling, and integrated studies in the Arase (ERG) project
}

\author{
Kanako Seki ${ }^{*} \mathbb{0}$, Yoshizumi Miyoshi ${ }^{2}$, Yusuke Ebihara ${ }^{3}$, Yuto Katoh ${ }^{4}$, Takanobu Amano ${ }^{1}$, Shinji Saito ${ }^{5}$, \\ Masafumi Shoji ${ }^{2}$, Aoi Nakamizo ${ }^{6}$, Kunihiro Keika' ${ }^{1}$ Tomoaki Hori ${ }^{2}$, Shin'ya Nakano ${ }^{7}$, Shigeto Watanabe ${ }^{8}$, \\ Kei Kamiya ${ }^{5}$, Naoko Takahashi ${ }^{1}$, Yoshiharu Omura ${ }^{3}$, Masahito Nose ${ }^{9}$, Mei-Ching Fok ${ }^{10}$, Takashi Tanaka ${ }^{11}$, \\ Akimasa leda ${ }^{2}$ and Akimasa Yoshikawa ${ }^{11}$
}

\begin{abstract}
Understanding of underlying mechanisms of drastic variations of the near-Earth space (geospace) is one of the current focuses of the magnetospheric physics. The science target of the geospace research project Exploration of energization and Radiation in Geospace (ERG) is to understand the geospace variations with a focus on the relativistic electron acceleration and loss processes. In order to achieve the goal, the ERG project consists of the three parts: the Arase (ERG) satellite, ground-based observations, and theory/modeling/integrated studies. The role of theory/modeling/integrated studies part is to promote relevant theoretical and simulation studies as well as integrated data analysis to combine different kinds of observations and modeling. Here we provide technical reports on simulation and empirical models related to the ERG project together with their roles in the integrated studies of dynamic geospace variations. The simulation and empirical models covered include the radial diffusion model of the radiation belt electrons, GEMSIS-RB and RBW models, CIMI model with global MHD simulation REPPU, GEMSIS-RC model, plasmasphere thermosphere model, self-consistent wave-particle interaction simulations (electron hybrid code and ion hybrid code), the ionospheric electric potential (GEMSIS-POT) model, and SuperDARN electric field models with data assimilation. ERG (Arase) science center tools to support integrated studies with various kinds of data are also briefly introduced.
\end{abstract}

Keywords: Inner magnetosphere, Numerical simulation/modeling, Geospace, Magnetic storms, Arase/ERG

\section{Introduction}

The largest disturbances in the near-Earth space (geospace) are called the magnetic storms or geospace storms, during which the development of the ring current, drastic variation of relativistic electrons in the radiation belts, and intense aurora activities occur (e.g., Williams 1987; Kamide et al. 1998; Baker et al. 2004). During the geospace storms, the relativistic electrons in the outer radiation belt may decrease during the main phase, while their variation in recovery phase depends on the storms (Reeves et al. 2003). The variation of relativistic electrons is determined by the competing effects of the acceleration and loss of the electrons. Various types of electron acceleration and loss processes have been proposed (e.g.,

\footnotetext{
*Correspondence: k.seki@eps.s.u-tokyo.ac.jp

${ }^{1}$ Graduate School of Science, University of Tokyo, Tokyo, Japan

Full list of author information is available at the end of the article
}

Friedel et al. 2002; Shprits et al. 2008a, b; and references therein). In order to understand the radiation belts variations, it is important to understand cross-energy coupling and cross-region coupling between plasmasphere, plasma sheet, ring current, and radiation belts, and magnetosphere-ionosphere coupling (Ebihara and Miyoshi 2011).

Since many of the acceleration and loss processes of the high-energy electrons are expected to be localized in terms of the local time (e.g., Summers et al. 1998; Ukhorskiy et al. 2009), it is important to assess effects of each process by simultaneous observations at different locations in geospace. The network of ground-based observations, which can observe electric and magnetic fields variations as well as particle precipitations globally at the foot points of the magnetic field lines connected to the geospace, play an important role in the geospace research (e.g., Shiokawa et al. 2017). In situ plasma observations by satellites, on 
the other hand, provide detailed information of the various types of wave-particle interactions. Particularly, observations near the magnetic equator are important for investigation of the relativistic electron variations (e.g., Seki et al. 2005). However, the numbers of observation points are limited. To supplement the limitation, numerical simulations/models are useful to investigate contribution of each candidate process quantitatively. There have been many efforts to simulate global variations of the inner magnetosphere (e.g., Ebihara and Ejiri 2003; Jordanova et al. 2010; Gkioulidou et al. 2011; and references therein). Therefore, the integrated studies combining both the ground-based and satellite observations with numerical simulations/ models are essential for understanding the dynamic variations of the geospace environment.

The Arase (ERG: Exploration of energization and Radiation in Geospace) satellite was launched in December 2016 as a part of the Japanese geospace research project, Exploration of energization and Radiation in Geospace (ERG) (Miyoshi et al. this issue, 2017). The science target of the ERG project is to understand underlying mechanisms of drastic variations of the geospace, such as magnetic storms, with a focus on the relativistic electron acceleration and loss processes in the terrestrial magnetosphere. In order to achieve the goal, the ERG project consists of three parts, i.e., the Arase (ERG) satellite, ground-based observations, and theory/modeling/integrated studies. The role of theory/modeling/integrated studies part is to promote related theoretical and simulation studies as well as integrated data analysis in combining different kinds of observations and modeling. From the planning phase of the ERG satellite, there have been many efforts to develop related simulation codes and new models.

In this paper, technical reports on simulation and empirical models related to the ERG project together with their roles in the integrated studies of dynamic geospace variations are provided. In "Simulation and empirical models related to the ERG project" section, characteristics and limitations of each simulation and empirical model are shown: "Radial diffusion model of the radiation belt electrons," "Relativistic guiding center test particle (GEMSIS-RB) model," "Comprehensive Inner Magnetosphere-Ionosphere Model (CIMI) with global MHD simulation REPPU," "Global drift-kinetic simulation of the ring current: GEMSIS-RC model," "Plasmasphere thermosphere model (PTM)," "Waveparticle interaction module for GEMSIS-RB (GEMSISRBW model)," "Self-consistent wave-particle interaction simulations," "A global ionospheric potential solver: GEMSIS-POT," and "Empirical ionospheric electric field models based on SuperDARN observations and data assimilation." In "ERG (Arase) science center tools" section, ERG (Arase) science center tools to promote integrated studies with various kinds of data are also briefly introduced. In "Discussions on roles of integrated studies" section, the role of these models/simulations in the integrated studies of the geospace variations is discussed, and "Summary" section provides a brief summary of this paper.

\section{Simulation and empirical models related to the ERG project \\ Radial diffusion model of the radiation belt electrons}

One of the processes to move trapped radiation belt particles across field lines is due to interactions with electric/magnetic field disturbances that are caused by MHD waves and other inductive fields (e.g., Elkington et al. 1999). Since the drifted particles interact with these waves many times, it is possible to consider these interactions as stochastic processes, and we can model these phenomena as radial diffusion. The diffusive process can be described using the following Fokker-Planck equation that describes time evolutions of the phase space density (e.g., Schulz and Lanzerotti 1974)

$$
\frac{\partial f}{\partial t}=L^{2}\left(\frac{\partial}{\partial L} \frac{D_{L L}}{L^{2}} \frac{\partial f}{\partial L}\right)+S-L
$$

where $f$ is the phase space density that is a function of the first and second invariants and $L$-shell. $D_{L L}$ is the radial diffusion coefficient, and $S$ and $L$ are source and loss, respectively. For the loss term, the lifetimes due to pitch angle scattering loss can be simply assumed, which depends on the $L$-shell and energy, while some studies developed three-dimensional diffusion model for not only radial diffusion but also pitch angle scattering and energy (momentum) diffusion in the velocity space, which shows wave-particle interactions as source and loss processes (e.g., Shprits et al. 2008a, b; Subbotin and Shprits 2009; Xiao et al. 2010).

The radial diffusion model has been used in many studies (Brautigam and Albert 2000; Miyoshi et al. 2003, 2004; Shprits et al. 2006). The model can capture several fundamental phenomena of the radiation belts. For example, the flux dropout due to outward diffusion (e.g., Brautigam and Albert 2000; Miyoshi et al. 2003; Shprits et al. 2006). The flux recovery and enhancement during the storm recovery phase were sometimes reproduced (e.g., Miyoshi et al. 2003; Shprits et al. 2009; Xiao et al. 2010). Recently, Van Allen Probes discovered three belt structures (Baker et al. 2013), and development of such spatial structure was reproduced by the radial diffusion model with the inclusion of wave-particle interactions (Shprits et al. 2013). Long-term variations for longer than a solar cycle are also reproduced by the radial diffusion model (Miyoshi et al. 2004; Maget et al. 2007).

The data assimilation is implemented in the radial diffusion model to estimate non-radial diffusion term 
(Koller et al. 2007; Kellerman et al. 2014) using the ensemble Kalman filter. The data assimilation should be important to improve the performance of the simulation as well as estimate the diffusion coefficients.

In ERG team, Nagoya University has developed the radial diffusion model and simulated the storm-time variations as well as the solar cycle variations (Miyoshi et al. 2003, 2004). This model includes the pitch angle scattering as lifetimes due to wave-particle interactions and Coulomb collisions besides the radial diffusion. The model uses several empirical radial diffusion coefficients as a function of geomagnetic indices and solar wind (e.g., Brautigam and Albert 2000; Li et al. 2001). The loss terms are described by the lifetimes due to Coulomb collisions and wave-particle interactions inside the plasmasphere. Three different wave sources are assumed inside the plasmasphere: plasmaspheric hiss, lightning whistler, and VLF transmitters. Using parameters for these wave sources given by Abel and Thorne (1998) and Albert (1999), we determined the lifetimes by wave-particle interactions with the same calculation method of Lyons et al. (1972) and Albert (1999).

The Arase satellite has continuously observed subrelativistic and relativistic electrons from $70 \mathrm{keV}$ to $20 \mathrm{MeV}$ by high-energy electron instruments HEP (Mitani et al. this issue 2017) and XEP (Higashio and Matsumoto, this issue 2017), and it is planned to compare Arase observations with the radial diffusion simulation. Since Arase satellite has measured several magnetic storms, it is interesting to compare the Arase observations with the simulation for several storms, which would be helpful to discriminate what physical process is dominant in causing the large flux enhancement (Miyoshi et al. this issue 2017). The data assimilation is also planned for investigating the radial diffusion coefficients and nondiffusive processes such as internal accelerations. Moreover, estimation of the radial diffusion coefficient is a key to improve the model performance. The electric fields measured by PWE/EFD (Kasaba et al. 2017) and magnetic fields measured by MGF (Matsuoka et al. this issue 2017) can be used to estimate the radial diffusion coefficient in the model. The radial diffusion model can be used for an assessment to identify the physical processes that produce the global variations of the outer radiation belt.

\section{Relativistic guiding center test particle model: GEMSIS-RB model}

The Geospace Environment Modeling System for Integrated Studies-Radiation Belts (GEMSIS-RB) code is a three-dimensional, relativistic, test particle simulation code that uses the relativistic guiding center equations developed by Brizard and Chan (1999). These equations solve trajectories of relativistic electrons in the three-dimensional electromagnetic fields using the fourth-order Runge-Kutta method. Test particles of electron conserve the first adiabatic invariant, so the electromagnetic fields should be in magnetohydrodynamics (MHD) regime. Therefore, arbitrary electromagnetic fields derived from models such as Tsyganenko 05 model (magnetic field only, Tsyganenko and Sitnov 2005), a global MHD model, and the GEMSIS-RC model ("Global drift-kinetic simulation of the ring current: GEMSIS-RC model" section, Amano et al. 2011) can be applied to the GEMSIS-RB model.

In the Tsyganenko 05 model and the global MHD model that produce the magnetopause boundary of the geomagnetic field, the GEMSIS-RB simulation demonstrates the magnetopause shadowing process (MPS) by calculating distance between the boundary and the guiding center positions. Figure 1 shows guiding center positions of $1 \mathrm{MeV}$ electrons with 90 degrees (top panel) and 10 degrees (bottom panel) pitch angles at the magnetic equator viewed from the dawnside in a storm-time geomagnetic field calculated from the Tsyganenko 05 model. Solid lines and gray circles show magnetic field lines and magnetopause boundary, respectively. Colored solid circles show the trajectories of 32 test electrons which have different starting points plotted every $5 \mathrm{~s}$ in $30 \mathrm{~min}$. The colors indicate the minimum distance between the position and the magnetopause boundary. In the case of $90^{\circ}$ pitch angle at the magnetic equator, the trajectories lean to the southern hemisphere. This is because of the drift shell bifurcation (DSB) where the drift shell of electrons is bifurcated on the dayside (e.g., Kim et al. 2008; Saito et al. 2010). These electrons approach to the magnetopause boundary on the dayside, so they are expected to be lost from the magnetosphere because of the magnetopause shadowing process. Note that in the case of a large dipole tilt angle, some electrons may not be lost even in the bifurcated orbit on the dayside (Saito et al. 2010). In the case of small pitch angle as shown in the bottom panel of Fig. 1, the drift shell is closer to the Earth than that of large pitch angle electrons on the dayside, because of the drift shell splitting (Sibeck et al. 1987). Only large pitch angle electrons are removed from the outer radiation belt in the solar wind dynamic pressure enhancement, which would be a cause of the butterfly distribution in pitch angle through the MPS. The RB model can demonstrate the MPS by calculating trajectories of electrons in timevarying geomagnetic field during the storm-time.

We use a particle weighting method in the GEMSIS-RB model to calculate the flux distribution in the electromagnetic fields. With the method, we estimate a particle weight of each test particle by comparing the initial flux with test particle distributions. The weight has the unit of 


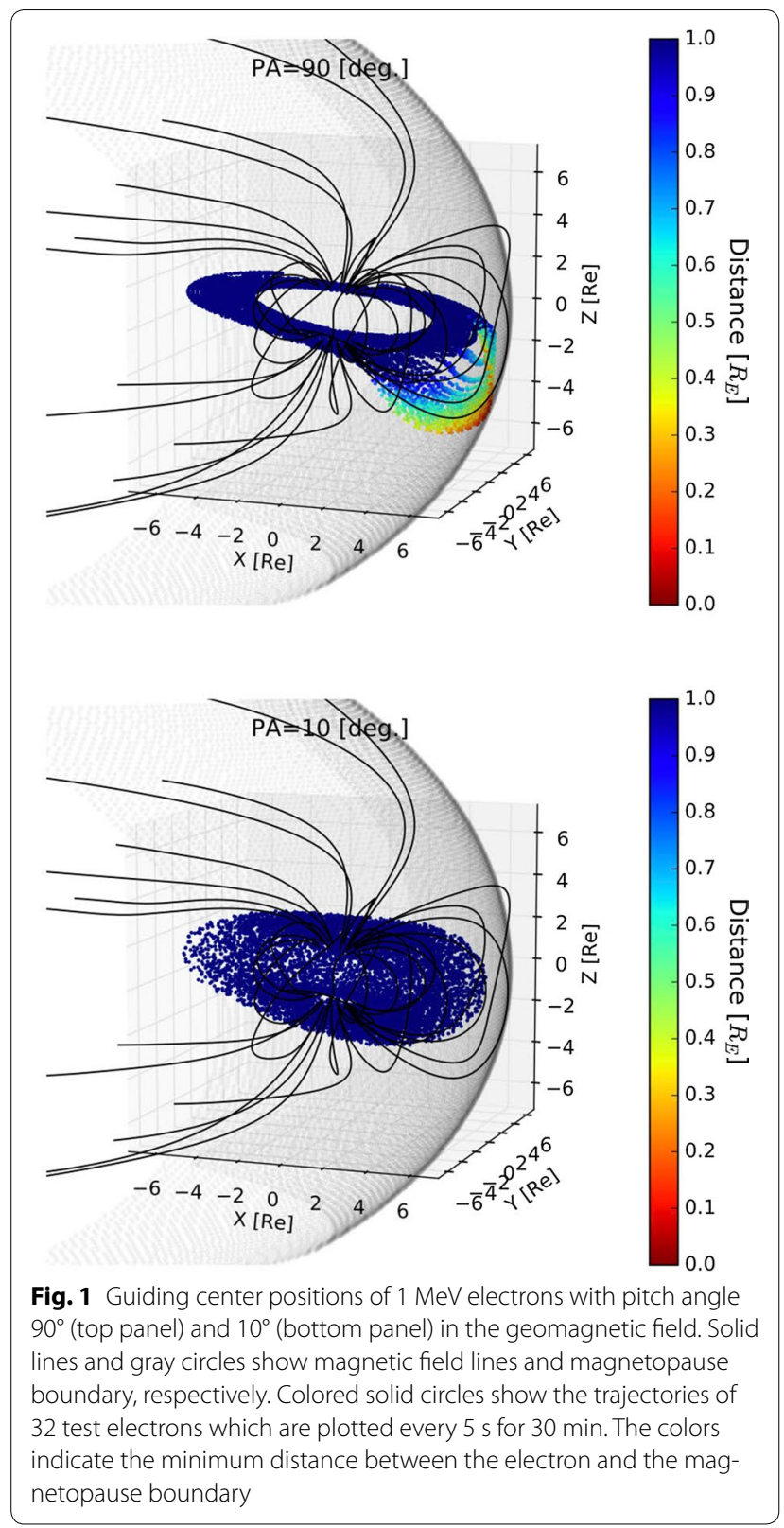

the number of the electrons, so it means that the weight of the test particle represents the number of electrons moving with the test particle. The GEMSIS-RB model calculates trajectories of the weighted particles and estimates flux distributions on a defined surface at any arbitrary position by counting the weighted particles passing through the surface.

Recent observations indicated evidences of electron acceleration by whistler-mode chorus waves that break the first adiabatic invariant (Miyoshi et al. 2003, 2007, 2013; Chen et al. 2007; Reeves et al. 2013). Therefore, the GEMSIS-RB model that conserves the first adiabatic invariant may not properly reproduce the flux variations when the whistler-mode chorus waves have an important role for the flux enhancement. In order to improve the model, we will apply wave-particle interaction model to the GEMSIS-RB model. In "Wave-particle interaction module for GEMSIS-RB (GEMSIS-RBW model)” section, we introduce the GEMSIS-RBW model that gives non-adiabatic momentum changes to the GEMSIS-RB model.

\section{Comprehensive Inner Magnetosphere-lonosphere Model (CIMI) with global MHD simulation REPPU}

Comprehensive Inner Magnetosphere-Ionosphere Model (CIMI) (Fok et al. 2014) solves the evolution of phase space density of electrons and ions as a function of the magnetic latitude $\lambda_{i}$, magnetic local time (MLT) $\phi_{i}$, the first adiabatic invariant $M$, and the second adiabatic invariant $K$. The bounce-averaged approximation (Roederer 1970) is used to describe motion of particles. The basic equation for CIMI is as follows

$$
\begin{aligned}
& \frac{\partial f}{\partial t}+\left\langle\frac{\mathrm{d} \lambda_{\mathrm{i}}}{\mathrm{d} t}\right\rangle \frac{\partial f_{\mathrm{s}}}{\partial \lambda_{i}}+\left\langle\frac{\mathrm{d} \phi_{\mathrm{i}}}{\mathrm{d} t}\right\rangle \frac{\partial f_{\mathrm{s}}}{\partial \phi_{i}} \\
& =\frac{1}{G} \frac{\partial}{\partial \alpha_{0}}\left[G\left(D_{\alpha_{0} \alpha_{0}} \frac{\partial f_{\mathrm{s}}}{\partial \alpha_{0}}+D_{\alpha_{0} E} \frac{\partial f_{\mathrm{s}}}{\partial E}\right)\right] \\
& +\frac{1}{G} \frac{\partial}{\partial E}\left[G\left(D_{E E} \frac{\partial f_{\mathrm{s}}}{\partial E}+D_{E \alpha_{0}} \frac{\partial f_{\mathrm{s}}}{\partial \alpha_{0}}\right)\right] \\
& -v\left\langle n_{\mathrm{H}}\right\rangle f_{\mathrm{s}}-\left(\frac{f_{\mathrm{s}}}{0.5 \tau_{\mathrm{b}}}\right)_{\text {Loss cone }}
\end{aligned}
$$

where $f_{\mathrm{s}}=f_{\mathrm{s}}\left(t, \lambda_{\mathrm{i}}, \phi_{\mathrm{i}}, M, K\right)$, the average distribution function between the mirror points of the field line with ionospheric foot point at $\lambda_{i}$ and $\phi_{i}$; $G=T\left(\alpha_{0}\right) \sin 2 \alpha_{0}\left(E+E_{0}\right) \sqrt{E\left(E+2 E_{0}\right)}, E$ is the kinetic energy, $E_{0}$ is the rest energy; $T$ is a function of equatorial pitch angle $\alpha_{0}$ (Fok et al. 2008); $D_{\alpha 0 \alpha 0}, D_{\mathrm{EE},} D_{\alpha 0 \mathrm{E},} D_{\mathrm{E} \alpha 0}$ are diffusion coefficients; $v$ is the speed of particle, $n_{\mathrm{H}}$ is the neutral hydrogen density; and $\tau_{\mathrm{b}}$ is the bounce period of the particle. The subscript $s$ denotes particle species, and the braces denote the bounce-averaged quantities. The left-hand side of the equation describes the advection due to drifts. The first two terms on the right-hand side mean the diffusion in energy and pitch angle space. The third term on the right-hand side is applied for ions, indicating the loss due to charge exchange with neutral hydrogen. The last term on the right-hand side implies the loss due to loss cone. The phase space density can be easily mapped to the coordinates specified by $L$ value, MLT, kinetic energy $E$ and equatorial pitch angle $\alpha_{0}$. The prime output of CIMI is the differential flux in the energy ranging from $1 \mathrm{keV}$ to $4 \mathrm{MeV}$, and pitch angle ranging from $0^{\circ}$ to $90^{\circ}$. The coordinate system is fixed at the 
ionosphere where the magnetic field is strong enough to consider that the induction electric field is negligible.

Non-dipole magnetic field models can be employed. The magnetic field model must satisfy the following conditions. Firstly, the magnetic field line must be closed. When the magnetic field line becomes open, we set the phase space density of the particles to be zero. Secondly, the number of minima of the magnetic field must be one along a field line. This requirement comes from the bounce-averaged approximation that assumes that all the trapped particles pass through the equatorial plane (minimum of the magnetic field). In the standard setting, the Tsyganenko magnetic field models are often chosen. The electric potential field is imposed on the ionosphere. In the standard setting, the Volland-Stern (Volland 1973; Stern 1975), or the Weimer (2001) convection electric field model can be used. The Weimer convection electric field model is an empirical model depending on the solar wind speed, density, and interplanetary magnetic field (IMF). In addition to the convection electric field, the corotation electric field is also imposed on the ionosphere. The CIMI is also capable of solving the ionospheric electric potential for given field-aligned currents (FACs) and ionospheric conductivities. The FACs are derived from the plasma pressure distribution (ring current) in the inner magnetosphere. The electric potential given by the empirical model is imposed on the poleward boundary of the ionospheric domain. An elliptic partial differential equation is solved to obtain the 2-dimensional distribution of the electric potential in the ionosphere. Based on the electric potential imposed on the ionosphere, the bounce-averaged drift velocity is calculated. In the standard setting, the Maxwellian distribution is imposed to the outer boundary of the magnetospheric domain. The number density, $N_{\mathrm{ps}}\left(\mathrm{cm}^{-3}\right)=0.395+0.025$ $N_{\mathrm{sw}}\left(\mathrm{cm}^{-3}\right)$ (Ebihara and Ejiri 2000), and the ion temperature, $T_{\mathrm{ps}}(\mathrm{keV})=-3.65+0.019 V_{\mathrm{sw}}(\mathrm{km} / \mathrm{s})$ (Borovsky et al. 1998), are used, where $N_{\mathrm{sw}}$ and $V_{\mathrm{sw}}$ are the solar wind density and the solar wind velocity, respectively. The plasmaspheric cold plasma density is also calculated for the given electric potential distribution in the ionosphere (Ober et al. 1997).

The CIMI can also be coupled with global magnetohydrodynamics (MHD) models (i.e., Glocer et al. 2013). In this subsection, we show an example of the simulation result of CIMI with the electric and magnetic fields provided by the MHD simulation REProduce Plasma Universe (REPPU) (Tanaka 2015). REPPU is capable of reproducing important features that manifest a substorm including quiet arcs, north-south-/east-west-aligned arcs and equatorward movement of the auroral oval during the growth phase, generation of FACs and westward traveling surge during the expansion phase, and overshielding (in which the polarity of the convection electric field in the inner magnetosphere is reversed) during the recovery phase (Ebihara et al. 2014; Ebihara and Tanaka 2015a, b, 2016; Tanaka 2015; Tanaka et al. 2017). In order to construct a steady-state magnetosphere, we imposed the following parameters on the upstream of the solar wind: solar wind speed of $372 \mathrm{~km} / \mathrm{s}$, solar wind density of $5 \mathrm{~cm}^{-3}$, IMF $B_{y}$ of $-2.5 \mathrm{nT}$, and IMF $B_{z}$ of $5 \mathrm{nT}$. Then, we changed the solar wind parameters to these values: solar wind speed of $700 \mathrm{~km} / \mathrm{s}$ and IMF $B_{z}$ of $-5 \mathrm{nT}$ (the solar wind density and IMF $B_{\mathrm{y}}$ remain the same). We defined " $T=0$ " at the moment when the southward IMF reached $X=40$ Re. At $T \sim 4 \mathrm{~min}$, the southward IMF reached the bow shock. The magnetospheric and ionospheric convection are enhanced because of the southward IMF. The polar cap potential drop reached $\sim 105 \mathrm{kV}$. At $T \sim 42 \mathrm{~min}$, a near-Earth neutral line appeared in the plasma sheet. At $T \sim 49 \mathrm{~min}$, the upward FACs started to increase on the nightside, and the auroral electrojet (ionospheric Hall current) started to intensify. We call this moment the onset of the first substorm expansion. Readers may refer Ebihara and Tanaka (2015a, b, 2016) for detailed sequence of the substorm (Fig. 2). Figure 6 is the same as Fig. 5 except for a different orbital phase of the virtual Arase. The virtual Arase proceeds that shown in Fig. 5 by 3 h. In general, the difference between Figs. 5 and 6 is basically caused by spatial variation, rather than temporal variation. The flux and the anisotropy are higher at higher $\mathrm{L}$ than at lower L. It can also be seen that how is the magnetic field line just before the onset of the first substorm expansion $(T \sim 49 \mathrm{~min})$. Subsequently, many onsets took place. As for CIMI, the Kappa

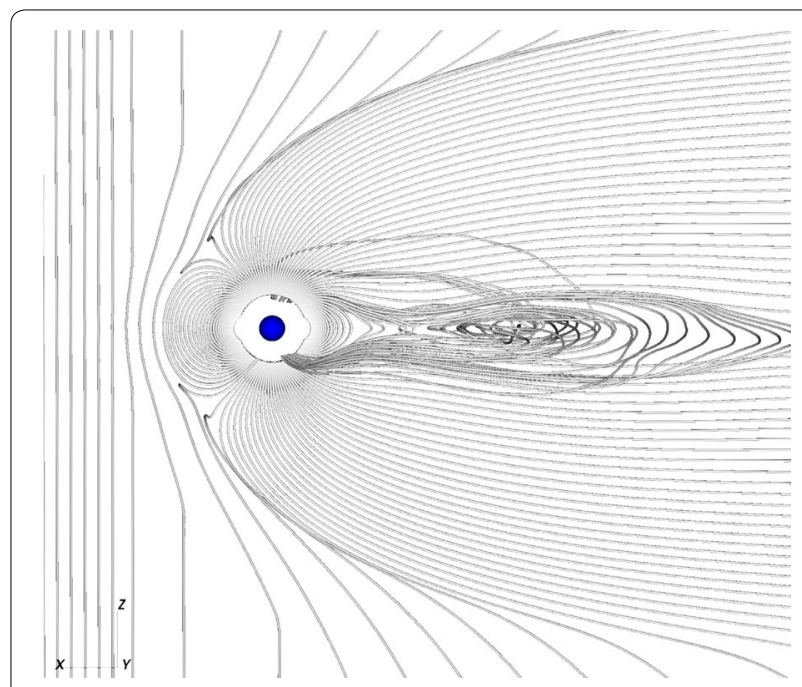

Fig. 2 Magnetic field lines just before the onset of the first substorm expansion onset. The sphere represents the Earth. The Sun is to the left 
distribution function of hot electrons was imposed on the outer boundary that is located on the magnetic field line extending from the magnetic latitude of $77^{\circ}$. The density and the equivalent temperature at the outer boundary were provided by REPPU. The temperature obtained by REPPU was divided by 7. The Kappa value is assumed to be a constant of 3.5. The initial condition for CIMI was provided by an empirical model of radiation belt electrons, the AE8MAX model (Gaffey and Bilitza 1994).

Figure 3 summarized the differential flux of the electrons from $\mathrm{keV}$ to $\mathrm{MeV}$ range at $T=169 \mathrm{~min}(2 \mathrm{~h}$ after the first onset). No diffusion was introduced in the simulation. Hot electrons have been subsequently injected into the inner magnetosphere due to enhanced convection and subsequent substorms. After being injected, the electrons drift eastward due to grad-B drift, curvature drift, and $\mathrm{E} \times \mathrm{B}$ drift. Consequently, the electron flux at $\mathrm{keV}$ and $10 \mathrm{~s}$ of $\mathrm{keV}$ ranges appears to increase on the morning side. No remarkable change is found at the $\mathrm{MeV}$ range compared to the low energies at this particular time. There have been studies to investigate electron temperature anisotropy and whistler-mode instability in simulations (e.g., Jordanova et al. 2012; and references therein). Figure 4 shows the temperature anisotropy ( $A$ $\equiv T_{\perp} / T_{\|}-1$, where $T_{\perp}$ and $T_{\|}$are the perpendicular temperature and parallel temperature, respectively), the ratio between the electron plasma frequency $\left(f_{\mathrm{pe}}\right)$ and the electron cyclotron frequency $\left(f_{\text {ce }}\right)$, the ratio between the hot electron density $\left(N_{\mathrm{H}}\right)$ and the cold electron density $\left(N_{\mathrm{c}}\right)$, and the cold electron density in the equatorial plane. The temperature anisotropy significantly increases at the leading edge of the population of newly injected
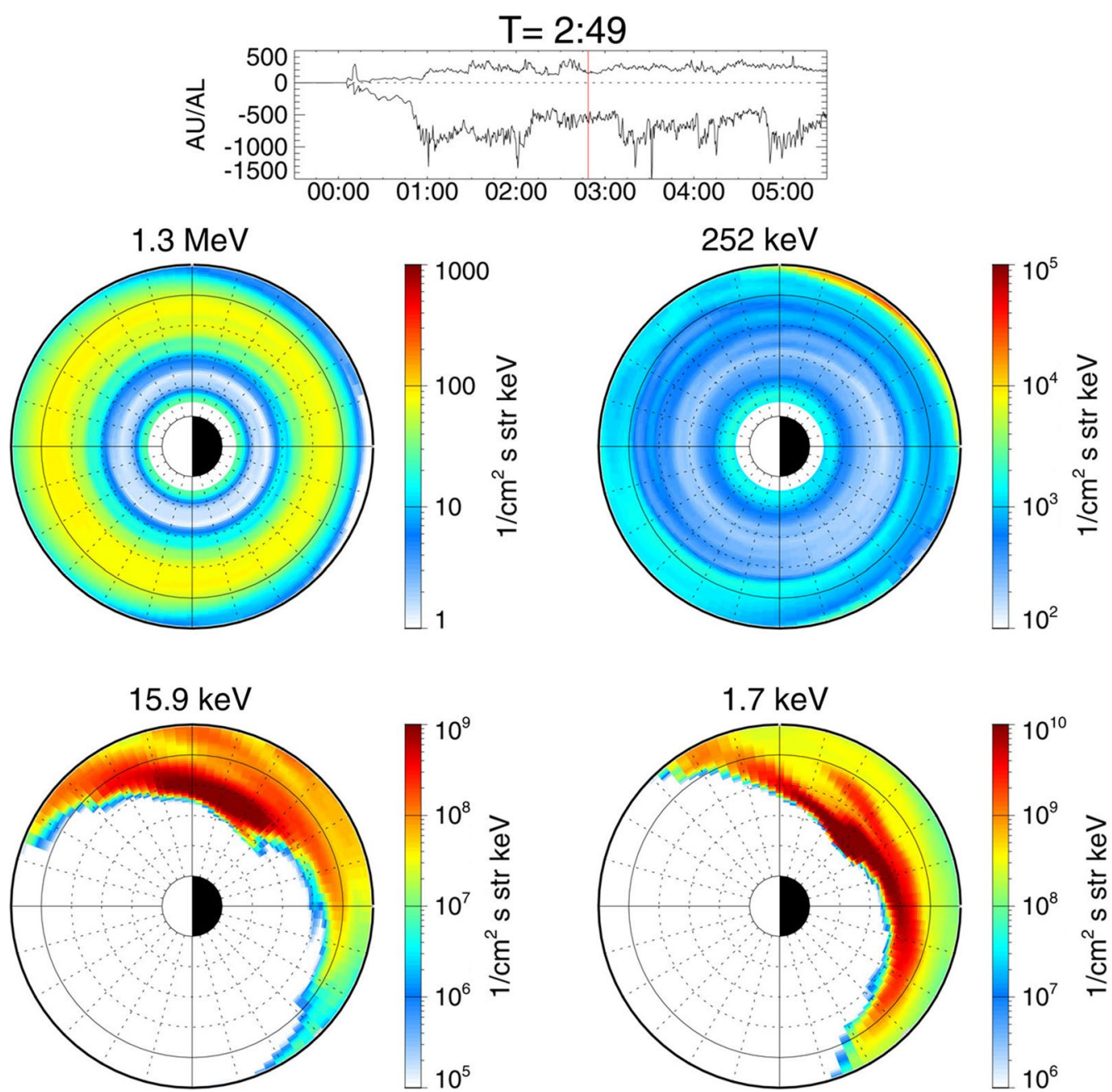

Fig. 3 Differential flux of electrons with equatorial pitch angle of $90^{\circ}$ with kinetic energy of 1.3 MeV, 252.0, 15.9 and 1.7 keV at $T=169$ min (2:49). The Sun is to the left. The outer circle corresponds to the geocentric distance of 6 Re. In the top panel, the calculated AU/AL indices are shown 


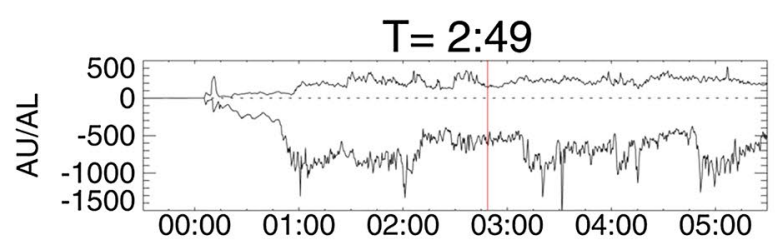

a Temperature anistropy

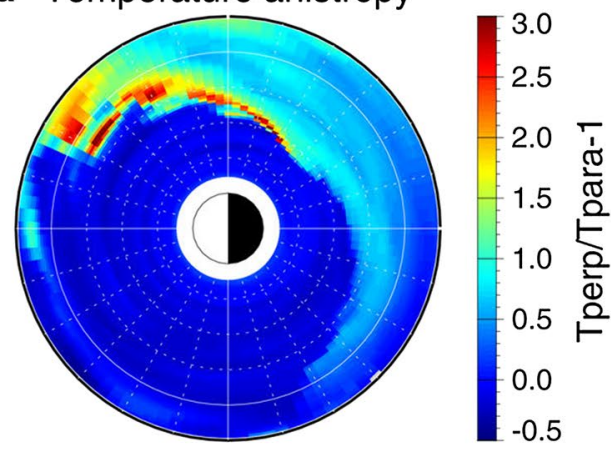

c

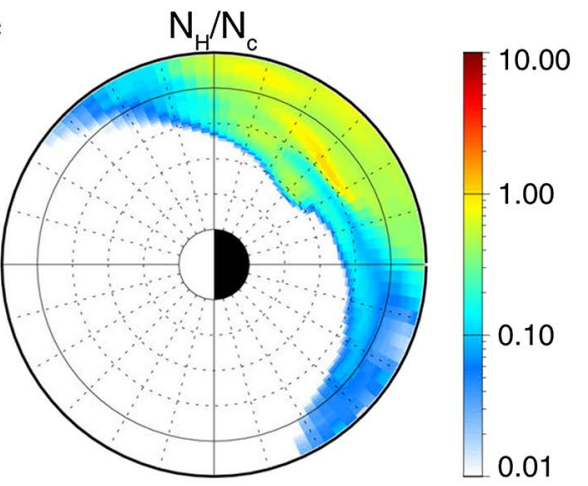

e
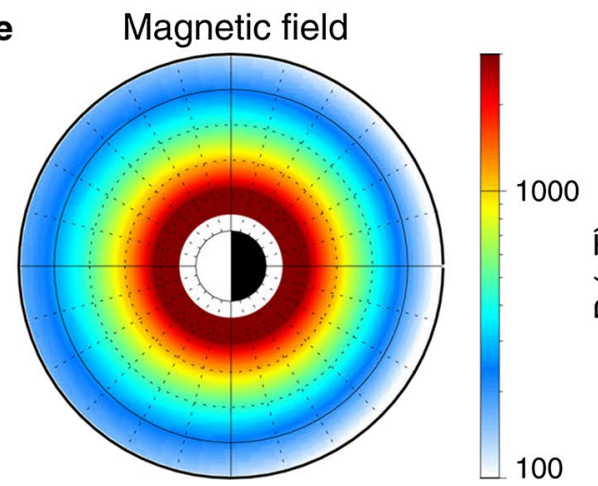

b

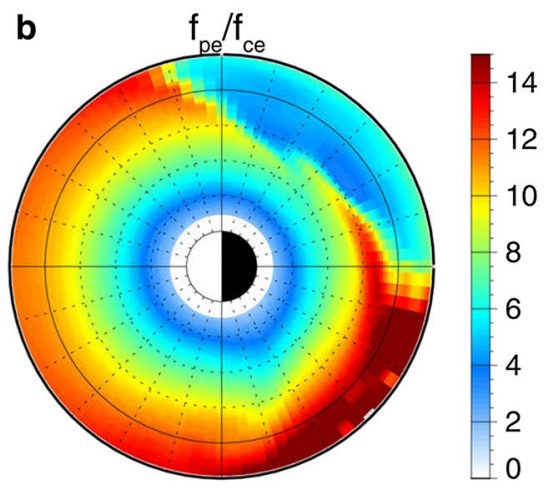

d Cold electron density

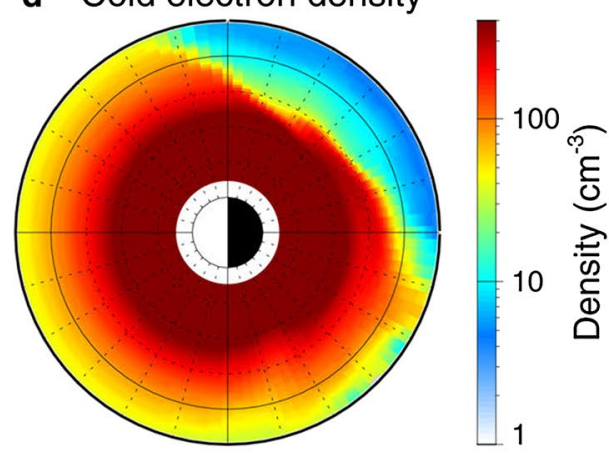

f

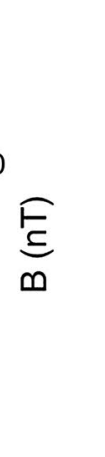

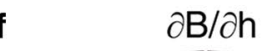

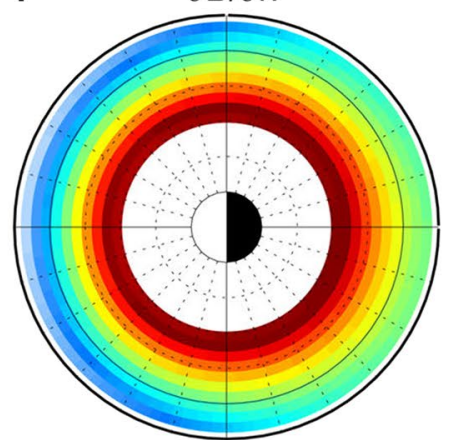

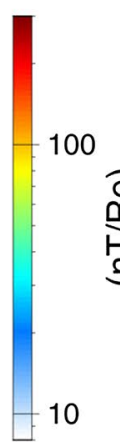

Fig. 4 a Temperature anisotropy $\left(T_{\perp} / T_{\|}-1\right.$, where $T_{\perp}$ and $T_{\|}$are the perpendicular and parallel temperature), $\mathbf{b}$ the ratio between the electron plasma frequency $\left(f_{\mathrm{pe}}\right)$ and the electron cyclotron frequency $\left(f_{\mathrm{ce}}\right)$, c the ratio between the hot electron density $\left(N_{H}\right)$ and the cold electron density $\left(N_{c}\right), \mathbf{d}$ the cold electron density in the equatorial plane, $\mathbf{e}$ the magnitude of the magnetic field, and $\mathbf{f}$ the gradient of the magnetic field along a field line at $T=169 \mathrm{~min}(2: 49)$. The Sun is to the left

hot electrons. These electrons originate in the nightside plasma sheet, drifting eastward. The $f_{\text {pe }} / f_{\text {ce }}$ ratio substantially decreases in the midnight-dawn sector. This is consistent with the statistically obtained distribution of the $f_{\text {pe }} / f_{\text {ce }}$ ratio (Meredith et al. 2003). The decrease in the $f_{\mathrm{pe}} / f_{\text {ce }}$ ratio results from the decrease in the cold electron 
density (erosion of the plasmasphere). The $N_{\mathrm{H}} / N_{\mathrm{c}}$ ratio is enhanced in the midnight-dawn sector due to injection of hot electrons. The $N_{H} / N_{\mathrm{c}}$ ratio has been suggested to be one of the controlling factors for the generation of whistler-mode chorus. The linear growth rate for whistler-mode waves increases with increasing $N_{\mathrm{H}} / N_{\mathrm{c}}$ ratio (Hikishima et al. 2009). Chorus emissions emerged from the band of whistler-mode waves generated through the instability driven by the temperature anisotropy when the wave amplitude exceeds the threshold amplitude (Omura et al. 2008, 2009). The coherent whistler-mode waves are shown to accelerate energetic electrons very efficiently (Omura et al. 2007). The cold electron density decreased in the midnight-dawn sector because the cold electrons were removed by the dawn-dusk convection electric field. It is expected that whistler-mode chorus wave may grow favorably in the midnight-dawn sector. The magnitude of the magnetic field is local-time-asymmetric. The dayside magnetosphere is compressed by the solar wind, whereas the nightside magnetosphere is stretched by the current sheet. The slope of the magnitude of the magnetic field along a field line from the equatorial plane $\partial B / \partial h$, where $h$ is a line element of a field line, was calculated. The slope is larger on the nightside than on the dayside. According to a hybrid simulation, the characteristics of the rising tone emissions of whistler-mode chorus waves depend on the inhomogeneity of the magnetic field $\partial B / \partial h$. Distinct chorus elements with rising tones occur favorably in the conditions where the inhomogeneity of the magnetic field is small (Katoh and Omura 2013). The energy gain of electrons interacting with whistler-mode wave packets is also shown to depend on the inhomogeneity of the magnetic field (Omura et al. 2007).

Figure 5 shows spectrograms of electron fluxes taken along an orbit of Arase. The apogee took place in the midnight-dawn sector, and the virtual orbit was based on the actual orbit in March 2017. The magnetic latitude is assumed to be zero for the sake of simplicity. At $T=00: 50$, the electron flux started to increases in association with the first expansion onset. This means that the virtual Arase encountered the injected hot electrons in association with the first expansion onset. The flux of the $10 \mathrm{keV}$ electrons with large pitch angles is larger than with small pitch angles. Both the spatial and temporal variations are included in the spectrograms. Figure 6 is the same as Fig. 5 except for a different orbital phase of the virtual Arase. The virtual Arase proceeds that shown in Fig. 5 by $3 \mathrm{~h}$. In general, the difference between Figs. 5 and 6 is basically caused by spatial variation, rather than temporal variation. The flux and the anisotropy are higher at higher $\mathrm{L}$ than at lower $\mathrm{L}$. It can also be seen that the flux at large pitch angles is higher than at small pitch
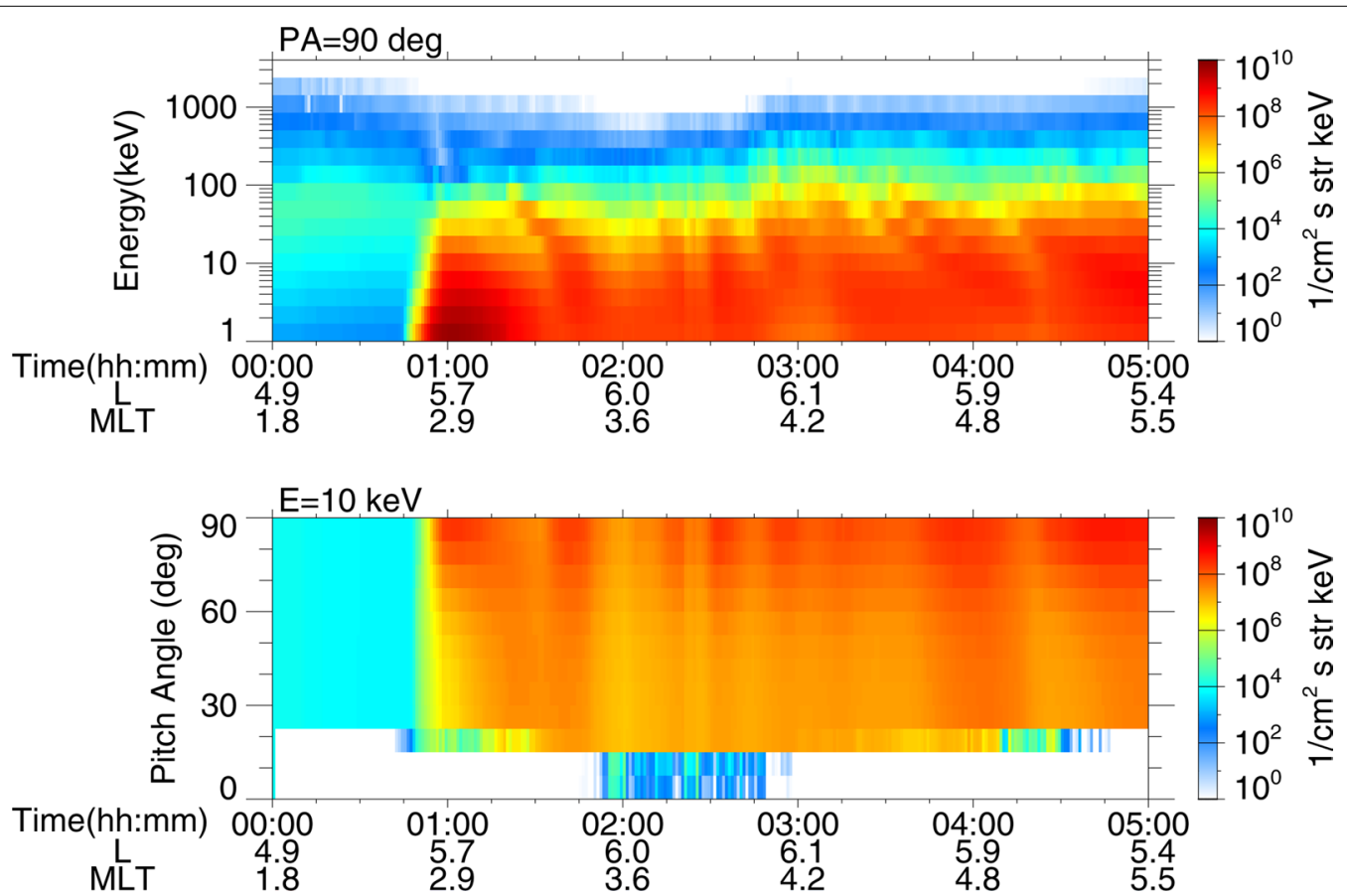

Fig. 5 Example of simulation results along the Arase trajectory: (top) energy versus time spectrogram of electrons with equatorial pitch angle of $90^{\circ}$, and (bottom) pitch angle versus time spectrogram of electrons with kinetic energy of $10 \mathrm{keV}$. The spectrograms are taken along a trajectory of Arase 


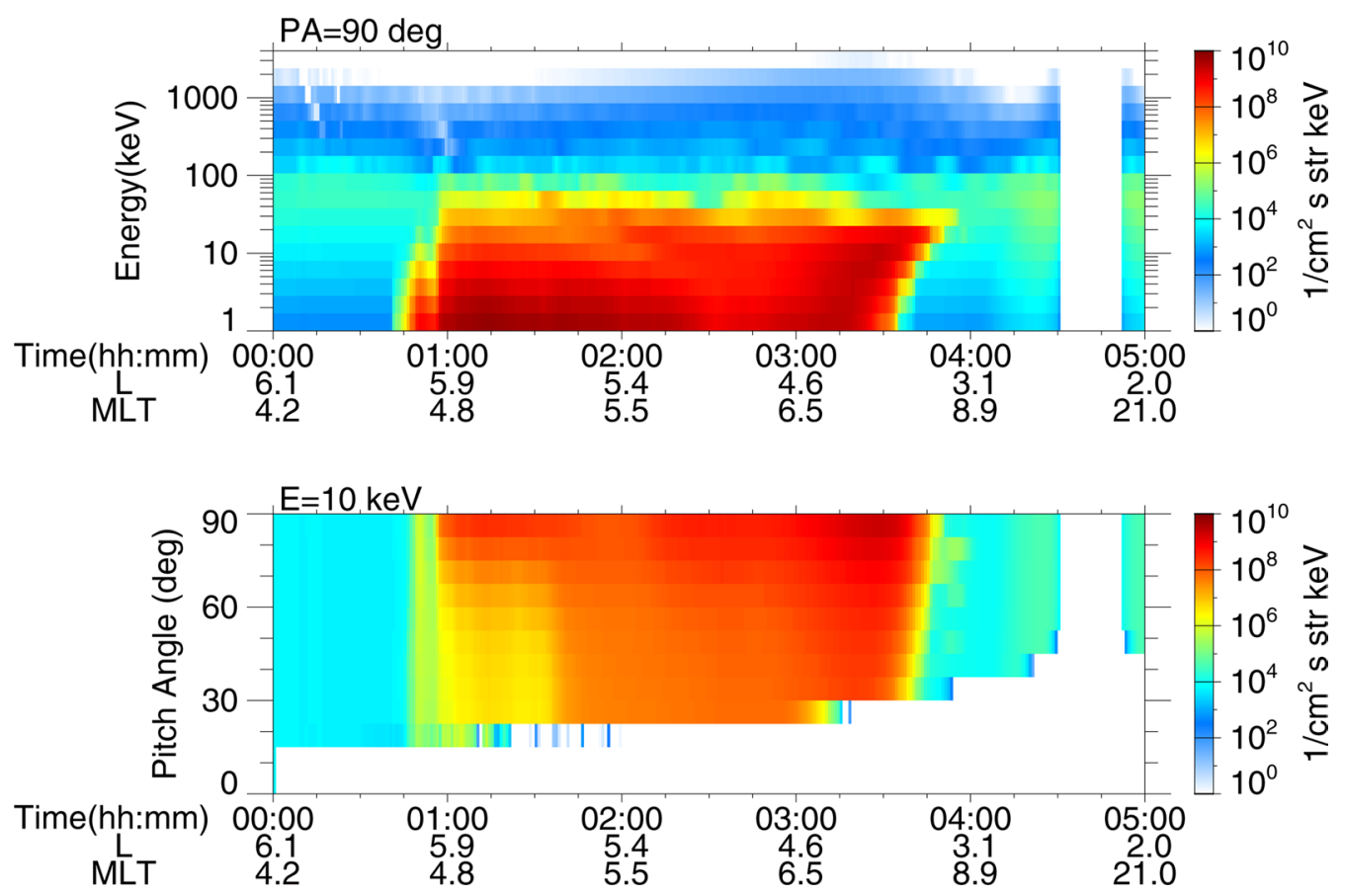

Fig. 6 Same as Fig. 5 except that Arase travels the same orbit $3 \mathrm{~h}$ earlier

angles because of pitch angle-dependent drift velocities of the electrons. In particular, the pitch angle anisotropy of the $10 \mathrm{keV}$ electrons is significantly large at the inner edge of the hot electron population in the inbound orbit. Consequently, the temperature anisotropy significantly increases on the dawnside as shown in Fig. 4a.

\section{Global drift-kinetic simulation of the ring current: GEMSIS-RC model}

It is important to follow the dynamics of the ring current particles and the electromagnetic field evolution in a fully self-consistent manner. In other words, the drift and bounce motions modify the particle pressure distribution, which produces an induction electric field and the magnetic field evolves according to Faraday's law. The modified electromagnetic field then, of course, affects the particle motion. This self-consistent coupling between the particles and field is not incorporated in popular ring current models that have been used in the inner magnetosphere community. Substantial effort has been devoted recently to combine a conventional ring current model with a global MHD simulation to take into account some sort of self-consistent couplings (e.g., De Zeeuw et al. 2004; Pembroke et al. 2012; Glocer et al. 2013). However, there still remain a lot of technical issues that are related to inconsistencies between models based on different physics. We believe that the fully self-consistent coupling may be incorporated only when both the particle and field dynamics are governed by the same physical principles.

The GEMSIS-RC model (Amano et al. 2011) had introduced this fully self-consistent coupling for the first time. This model solves the drift-kinetic equation and Maxwell's equations at the same time with appropriate coupling between the two. Since the use of the drift-kinetic equation means that the gyrophase dependence of the phase space density is ignored, the distribution function in a reduced five-dimensional phase space must be solved. Once the distribution function is obtained, the current density can be calculated using the moment quantities, which is then substituted into Maxwell's equations.

It is important to note that the model properly takes into account propagation of MHD waves both transverse and parallel to the magnetic field. Therefore, one may model a short timescale transient phenomenon involving MHD wave propagation. Also, it takes into account the particle bounce motion along a magnetic field line. This makes it an ideal tool to simulate (both internally and externally driven) ULF wave phenomena. On the other hand, the model clearly requires a lot more computational resources than conventional approaches as it solves the five-dimensional phase space. Therefore, we may consider the model as being complementary to other 
ring current models which require less computational resources.

Figure 7a shows a snapshot of the electric field distribution obtained from the Pc5 ULF (ultra-low frequency) wave simulation in the inner magnetosphere with the GEMSIS-RC model. A monochromatic poloidal Pc5 wave with the period of $300 \mathrm{~s}$ and azimuthal wave number of 2 is imposed at the outer simulation boundary at $L=6.6$. Then, the Pc5 wave propagates self-consistently in the 3-D space in the inner magnetosphere with some interaction with the ring current ions. One of important applications of the ULF simulations is to investigate the radial transport process of relativistic electrons with the Pc5 waves. Figure $7 \mathrm{~b}, \mathrm{c}$ shows the result of a combined simulation between the GEMSIS-RC and GEMSISRB (see "Relativistic guiding center test particle model: GEMSIS-RB model" section) models. By calculating radial transport of relativistic electrons in the 3-D electromagnetic fields under the monochromatic Pc5 wave obtained from GEMSIS-RC, we can investigate the equatorial pitch angle dependence of the radial transport of radiation belt electrons due to the Pc5 ULF waves. In this combined simulation, we set a sharp PSD (phase space density) gradient of the relativistic electrons at $L=6$ as an initial condition. The electrons initially have isotropic pitch angle distributions. Then, the trajectories of many electrons are traced under existence of monochromatic Pc5 waves for about 10 wave periods. As shown in
Fig. 7b, c, the electrons are transported inward through interaction with the monochromatic Pc5 waves after the 10 wave periods. The structuring and peak formation at certain $\mathrm{L}$ in Fig. $7 \mathrm{~b}$ for electrons with the pitch angle of 90 degrees are consistent with previous studies (e.g., Degeling et al. 2008). The result also shows that the radial transport rate is different between the perpendicular (Fig. 7b) and oblique (Fig. 7c) pitch angle electrons. Comparison of these simulation results with the Arase and other satellite observations will allow us to investigate the radial transport acceleration of relativistic electrons quantitatively.

\section{Plasmasphere thermosphere model (PTM)}

The plasmasphere is an inner part of the Earth's magnetosphere. There is dense and cold plasma (a few eV) generated in the Earth's ionosphere. The electrons in the ionosphere move along the Earth's magnetic field lines and the electrons with the energy larger than the escape energy can reach the inner magnetosphere, though the ions cannot move to the inner magnetosphere because of heavy mass. Therefore, an electric field develops in the region between the ionosphere and the inner magnetosphere. By the generated electric field, the ionospheric ions begin to move upward. The ionospheric electrons and ions move to the inner magnetosphere together. The light ions such as $\mathrm{H}+, \mathrm{He}+$, and $\mathrm{O}+$ can easily escape from the ionosphere. Within a period from a few hours

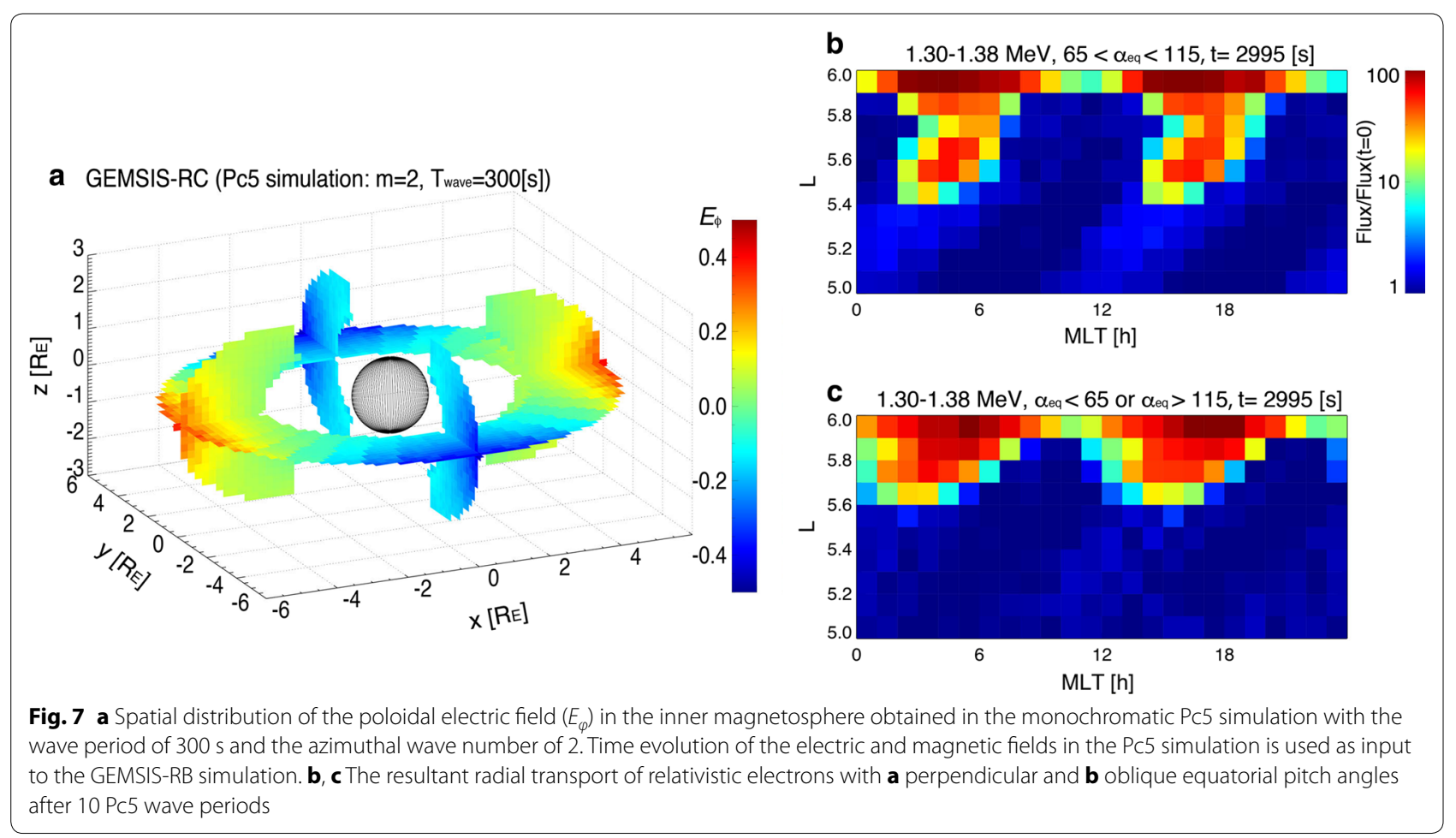


to days, the escaped plasma accumulates until an equilibrium state is reached. The plasmasphere nearly rotates with the Earth. The region changes in size with the geomagnetic activity, so that the loss of the plasmaspheric plasma and/or the refilling of the plasmasphere depends on the geomagnetic activity.

There are two types of plasmaspheric model. One is the model based on satellite data or ground-based observation data, and the other is physical model. Carpenter and Anderson (1992) developed an empirical model of equatorial electron density in the range of $2.25<L<8$ from sweep frequency receiver (SFR) radio measurements by International Sun-Earth Explorer (ISEE) 1 satellite. O'Brien and Moldwin (2003) developed an empirical model of the plasmapause location as a function of $\mathrm{Kp}, \mathrm{AE}$, and Dst with Combined Release and Radiation Effects Satellite (CRRES) observations. The global core plasma model (GCPM) is based on the data from Dynamics Explorer (DE) and ISEE satellites (Gallagher et al. 2000). The Akebono model was developed from Akebono satellite measurements, which includes electron temperature distributions at $1000-10,000 \mathrm{~km}$ altitudes (Kutiev et al. 2004). The IMAGE Model comes from Radio Plasma Imager (RPI) measurements from IMAGE. The model gives density distribution as a function of $L$ value and latitude (Huang et al. 2004).

Global Plasma Ionosphere Density (GPID) model is a physical model to estimate ion density and electron temperature in the plasmasphere along a magnetic flux tube (Webb and Essex 2001). The model gives $\mathrm{O}+$ and $\mathrm{H}+$ densities and temperatures depending on the solar and magnetic activities. The Sheffield University Plasmasphere Ionosphere Model (SUPIM) estimates the densities, field-aligned fluxes, and temperatures of $\mathrm{O}+, \mathrm{H}+$, $\mathrm{He}+, \mathrm{N} 2+, \mathrm{O} 2+$, and $\mathrm{NO}+$ ions, and electrons with time-dependent equations of continuity, momentum, and energy balance along eccentric dipole magnetic field lines (Bailey et al. 1997). Field Line Interhemispheric Plasma (FLIP) model solves the equations of continuity and momentum and energy conservation for $\mathrm{O}+, \mathrm{H}+, \mathrm{He}+$, and $\mathrm{N}+$ ions along the inclined dipole geomagnetic field lines (Tu et al. 2003).

We have developed a physical model Plasmasphere Thermosphere Model (PTM) to estimate ion and electron densities, temperatures, and velocities with time-dependent equations of continuity, momentum, and energy along dipole magnetic field lines at altitudes between $90 \mathrm{~km}$ and $10 \mathrm{Re}$. The equations are solved for $\mathrm{N} 2+$, $\mathrm{O} 2+, \mathrm{NO}+, \mathrm{O}+, \mathrm{He}+$, and $\mathrm{H}+$ in the ionosphere and the plasmasphere. We used the energy equations for the calculations of parallel and perpendicular temperatures of each ion species and electron to the local geomagnetic field lines. The two components of plasma temperature are important to describe the ion and electron heating and dynamics of plasma in the auroral latitude ionosphere, because the plasma is heated by auroral particle precipitation, auroral electric fields, and wave-particle interaction near the cusp region. The two component energy equations are also important to the dynamics of polar wind in the topside polar ionosphere and plasmasphere. The PTM includes low-latitude electric field model (Scherliess and Fejer 1999) and high-latitude electric field model associated with the magnetosphere-solar wind interaction (Volland 1973). The neutral atmosphere in the thermosphere is calculated for densities, temperatures, and velocities of $\mathrm{N} 2, \mathrm{O} 2, \mathrm{O}$, and $\mathrm{H}$ with equations of continuity, momentum, and energy. Since the ion heating in the auroral region is important to refill the plasmasphere, we constructed an empirical model of ion outflow, which is based on the observation data from Suprathermal Ion Massspectrometer (SMS) on Akebono satellite (Watanabe et al. 1992; Abe et al. 1993).

The PTM, thermosphere model, and ion outflow model are made as functions of time, season, longitude, latitude, altitude, solar activity, and geomagnetic activity. These models are solved simultaneously. The input parameters to the models are solar and geomagnetic activities only. The boundary conditions are set at the altitude of $90 \mathrm{~km}$, where we use the data from the mass spectrometer and incoherent scatter (MSIS) model (Hedin 1991). The electron densities by the PTM show clearly a density gradient change at altitude of $\sim 1500 \mathrm{~km}$ in the polar region and plasmapause. The density gradient change at $\sim 1500 \mathrm{~km}$ altitude is corresponding to the transition height from $\mathrm{O}+$ to $\mathrm{H}+$. The variations of plasmapause location and plasma density and temperature within the plasmasphere, and the generation of plasma tail during geomagnetic storms and the refilling of plasmasphere are reproduced. The obtained model results give a reference to understand conjunction observations between the Arase satellite and related ground-based observations. Coupling with an ionospheric electric field model based on data assimilation described in "Empirical ionospheric electric field models based on SuperDARN observations and data assimilation" section is also planned. The obtained plasmapause locations will be compared with the plasmapause observations by the Arase satellite to improve the model verification. The model is open to public in the web of "http://www.ep.sci.hokudai. ac.jp/ shw/space.html."

\section{Wave-particle interaction module for GEMSIS-RB (GEMSIS-RBW model)}

The GEMSIS-RBW (GEMSIS-RB: wave-particle interaction module) model (RBW model) calculates the non-adiabatic momentum changes in electrons within simulated 
whistler-mode chorus elements propagating along a magnetic field line. Bounce motion of electrons conserving the first adiabatic invariant of electrons and propagation of the waves along the field line are solved simultaneously in the RBW model. The adiabatic momentum change $\Delta \mathbf{P}_{\mathrm{a}}$ associated with the bounce motion and the non-adiabatic momentum change $\Delta \mathbf{P}_{\mathrm{w}}$ associated with the scattering by whistler chorus waves lead to the full momentum change $\Delta \mathbf{P}$ for a time step $\Delta t$

$$
\Delta \mathbf{P}=\Delta \mathbf{P}_{\mathrm{a}}+\Delta \mathbf{P}_{\mathrm{w}} .
$$

The full momentum change updates the momentum at the next time step.

While the adiabatic bounce motion calculates the guiding center position along the field line, the calculation for the non-adiabatic momentum changes in electrons is carried out when the guiding center of electron is in the same place as the whistler-mode chorus element. The RBW model simulates a discrete whistler-mode chorus element as a series of point packets. These packets each keep their wave frequency and amplitude. Wave number of each packet is derived from linear dispersion relation of whistler-mode wave using the background plasma density and magnetic field intensity at the packet's position. These packets each propagate at their own group velocity along the field line. Figure 8 shows an example of results calculated by the RBW simulation. Figure $8 \mathrm{a}$, b shows snap shots of whistler chorus elements propagating along the magnetic field line at $t=6.1$ and $6.3 \mathrm{~s}$. The solid line and color of solid circles show the magnetic field line and frequency of point packets of the applied chorus element, respectively. Chorus elements are launched following an applied dynamic spectrum as shown in Fig. 8d and propagate along the field line in both the northern and southern directions symmetrically. The calculation for particle scattering is carried out when the electron is within a whistler chorus element. Wave parameters at the electron position, which are wave frequency, wave number, and wave amplitude, are interpolated from the nearest two point packets in the chorus element.

The non-adiabatic momentum change due to whistlermode chorus is obtained by using the equation of motion for charged particle in the electromagnetic fields

$$
\frac{\mathrm{d} \mathbf{p}_{\boldsymbol{e}}}{\mathrm{d} t}=q_{\mathrm{e}}\left(\delta \mathbf{E}+\mathbf{v}_{\boldsymbol{e}} \times\left(\mathbf{B}_{\boldsymbol{o}}+\delta \mathbf{B}\right)\right)
$$

where $\mathbf{p}_{\boldsymbol{e}}$ is the electron momentum, $q_{\mathrm{e}}$ is the electron charge, $\mathbf{v}_{\boldsymbol{e}}$ is the electron velocity, and $\mathbf{B}_{\boldsymbol{o}}$ is the background magnetic field. The electric and magnetic fluctuations $\delta \mathbf{E}$ and $\delta \mathbf{B}$ with angular wave frequency $\omega$ and wave number parallel to the background magnetic field $k_{\|}$satisfy whistler dispersion relation. The equation of motion is solved by using the Boris method that is well used in
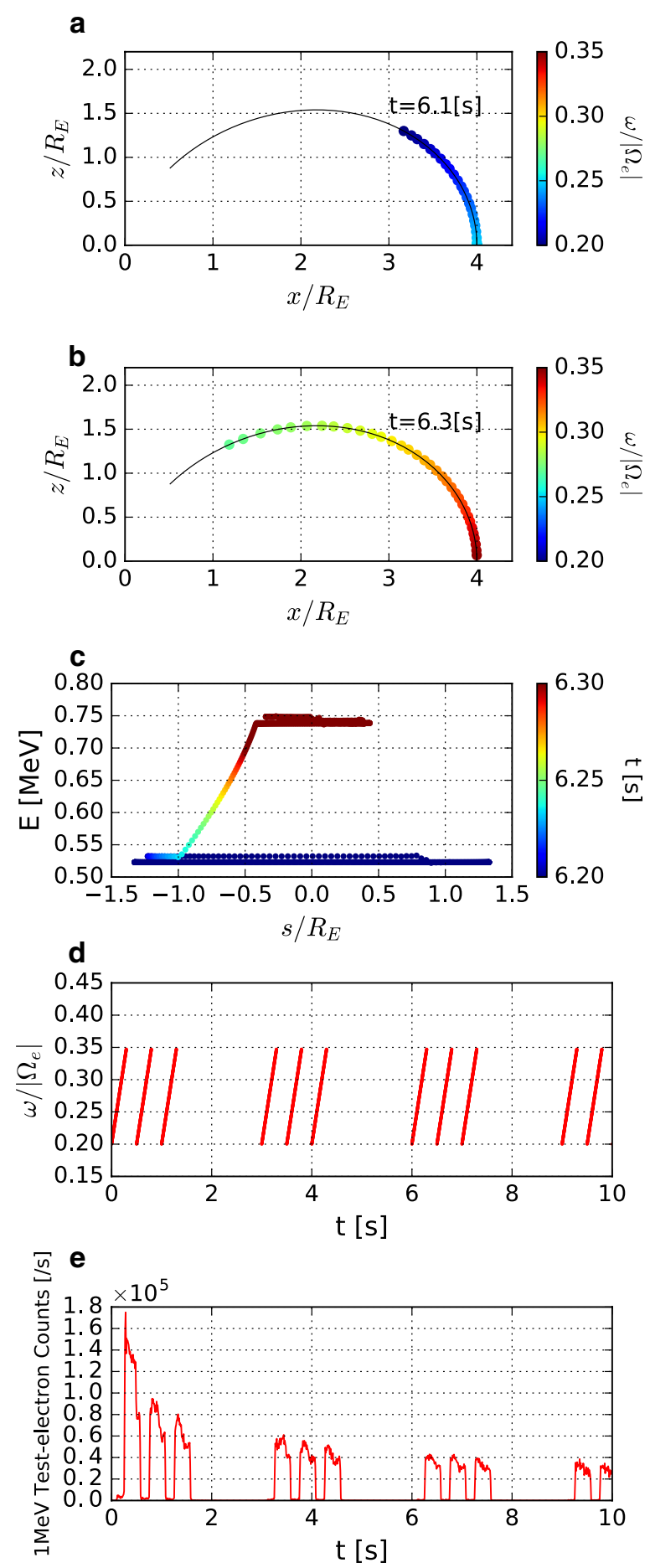

Fig. 8 A calculation example of GEMSIS-RBW. a, b Snapshots of whistler-mode chorus waves propagating along the magnetic field line (solid line) at $t=6.1$ and $6.3 \mathrm{~s}$. The color of solid circles shows frequency of wave packet. c Trajectory of an electron during $t=5$ and $t=7 \mathrm{~s}$ in $(s, E)$ plane, where $s$ is distance from the equator along the magnetic field line and $E$ is kinetic energy. The color of solid circles shows the time between 6.2 and 6.3 s. d Applied dynamic spectrum of whistler chorus elements. e Temporal variation of counts of $1 \mathrm{MeV}$ electrons precipitated into the atmosphere (100 km altitude) 
particle-in-cell method. The equation of motion derives the non-adiabatic momentum change $\Delta \mathbf{P}_{\mathrm{w}}$ in $\Delta t$, by resolving the electron cyclotron motion with $\delta t(\ll \Delta t)$. In order to solve the equation, it is necessary to derive the relative phase between the electron gyration and circularly polarized electromagnetic fluctuations. The relative phase $\zeta$ between the vector of magnetic field fluctuation and the electron gyration phase is estimated from

$$
\frac{\mathrm{d} \zeta}{\mathrm{d} t}=\frac{\mathrm{d} \phi_{\mathrm{e}}}{\mathrm{d} t}-\left(\omega-k_{\|} v_{\|}\right)
$$

where $\phi_{\mathrm{e}}$ is the electron gyration phase. The relative phase of electrons is updated, while electrons are in the whistler chorus element. Here we presume coherent whistler waves to calculate the relative phase from the equation.

The RBW simulation can calculate electron precipitation into the atmosphere by counting the number of electrons scattered into the loss cone. Figure 8e shows the temporal variation of the counts of precipitating electrons with energy of $1 \mathrm{MeV}$. These electrons are precipitated into the atmosphere (100 km altitudes assumed) by whistler chorus elements with wave magnetic amplitude of 200 pT propagating parallel to the background magnetic field away from the equator. The relativistic electron precipitation shows intermittent bursts that correspond to the emission of whistler chorus elements. Relationship between whistler chorus elements and relativistic electron precipitation is discussed in Saito et al. (2012). They have demonstrated that whistler-mode chorus waves propagating to high magnetic latitudes cause relativistic electron microbursts (e.g., Lorenzten et al. 2001). Miyoshi et al. (2015) estimated energy spectrum of precipitated electron flux, which is likely to be a source of pulsating aurora, by using the particle weighting method as used in the RB model. They showed evidence that a cause of pulsating aurora is whistler-mode chorus by comparing the RBW simulation data with the groundbased observations.

The RBW simulation can demonstrate not only atmospheric precipitation of radiation belt electrons but also relativistic electron acceleration by using the RBW model. Figure $8 \mathrm{c}$ shows a trajectory of an electron. Horizontal and vertical axes are distances from the magnetic equator along the field line and kinetic energy of the electron. Colors show the time at the electron position. The electron goes through the mirror motion at $t<6.2 \mathrm{~s}$ without any remarkable energy gain and loss, but it rapidly increases its kinetic energy at $t>6.2 \mathrm{~s}$. The energy gain is about $200 \mathrm{keV}$ in a short time which is of the order of $100 \mathrm{~ms}$. The acceleration is caused by nonlinear phase trapping (Albert 2002; Omura et al. 2007; Bortnik et al. 2008). Since the rapid acceleration by nonlinear phase trapping occurs in a coherent whistler-mode chorus, the acceleration process is completely different from quasilinear diffusion process which assumes statistical random phase of the waves. Saito et al. (2016) had done the RBW simulations to study influence of the nonlinear phase trapping on flux enhancement of relativistic electrons. They demonstrated that the nonlinear acceleration process, such as the phase trapping (e.g., Omura et al. 2007), causes the rapid flux enhancement of radiation belt electrons that is more efficient than quasilinear diffusion process. The RBW simulations suggested that quasilinear diffusion process is not always valid to fully describe the acceleration of relativistic electrons.

The GEMSIS-RBW model demonstrates particle scattering in whistler-mode chorus elements in the magnetic field line by solving the equation of motion directly. As shown in Fig. 8, the RBW simulation can resolve both acceleration and precipitation loss of radiation belt electrons by applying a dynamic spectrum of whistler chorus elements. The RBW simulation with a huge number of test electrons can demonstrate temporal variation of flux distributions in pitch angle and energy using particle weighting method as used in the RB model. Flux distributions and dynamic spectrum of whistler chorus waves observed by Arase and/or other satellites can be reproduced in the RBW simulation. Applying the dynamic spectrum and the particle weight to estimate the flux distribution, the RBW simulation can demonstrate temporal variation of the flux distributions associated with interactions between whistler chorus elements and radiation belt electrons.

By comparing the simulation data resolving acceleration and precipitation loss of radiation belt electrons with observation data by Arase, we expect progress in the study of wave-particle interactions in the radiation belt. In the future, the RBW model will be applied to a three-dimensional radiation belt model, like the GEMSIS-RB model. We couple the RB model with the RBW model by applying the non-adiabatic momentum change calculated from the RBW model to the RB model. The RBW model imports magnetic field data along a finitesize magnetic flux tube and momentum and position data of electrons in the flux tube from the RB model. Using these data, the RBW model calculates the nonadiabatic momentum changes with an applied dynamic spectrum of whistler chorus elements defined in the flux tube. The RBW model can be coupled with the RB model by applying the non-adiabatic momentum changes for the RB model. The next-generation RB model that incorporates the RBW model will have an important impact on the global radiation belt modeling that is expected to improve prediction of variation in radiation belt electron flux. 


\section{Self-consistent wave-particle interaction simulations}

Self-consistent simulations are used for studies of waveparticle interactions occurring in the inner magnetosphere. Results of self-consistent simulations contribute to the understanding of physical meaning of in situ but spatially sparse satellite observation data, as shown in Fig. 9. Self-consistent simulations reveal both spatial and temporal evolutions of plasma wave spectra, velocity distribution functions of energetic particles, and the waveparticle interactions. We can conduct direct comparison of simulation results with Arase satellite observations by employing observed parameters for the initial conditions of simulations. We discuss both electron hybrid and ion hybrid codes for the study of whistler-mode chorus and electromagnetic ion cyclotron waves occurring in the equatorial region of the inner magnetosphere.

\section{Electron hybrid code}

Electron hybrid code solves the evolution of electromagnetic field by Maxwell's equations together with the motion of energetic electrons by a standard particle-incell method with fully relativistic effects and the motion of cold electrons by the equation of motion of fluid (Katoh and Omura 2004). Ions are treated as an immobile

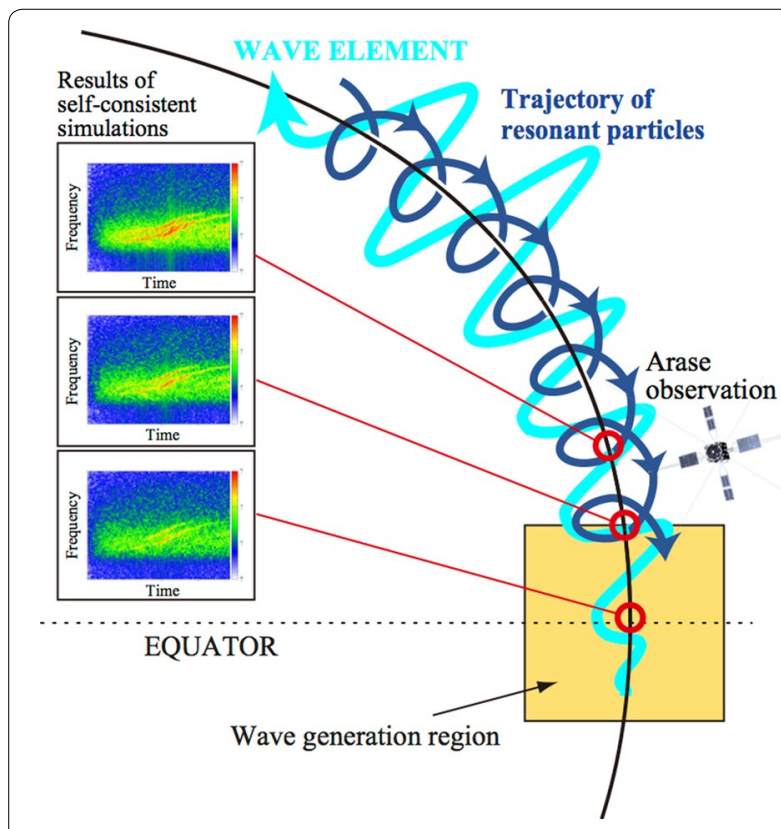

Fig. 9 Schematic diagram of roles of self-consistent simulations in studies of wave-particle interactions occurring in the equatorial region of the inner magnetosphere. Results of self-consistent simulations include both spatial and temporal evolutions of plasma wave spectra, velocity distribution functions of energetic particles, and their interactions. Self-consistent simulations contribute to the understanding of physical meanings of in situ but spatially sparse satellite observations background in the simulation system. By employing a spatially one-dimensional simulation system along a magnetic field line, Katoh and Omura (2007a) reproduced the generation of whistler-mode chorus emissions with rising tones. Rapid acceleration of relativistic electrons nonlinearly trapped by the generated chorus emissions has also been reproduced in the simulation (Katoh and Omura 2007b). Properties of the chorus generation have been investigated for the number density of energetic electrons (Katoh and Omura 2011), background magnetic field inhomogeneity (Katoh and Omura 2013), and temperature anisotropy of energetic electrons (Katoh et al. 2018b) by using the electron hybrid code. Simulation results have been compared with theoretical estimations of the threshold (Omura et al. 2009) and optimum wave amplitudes (Omura and Nunn 2011) for the generation of chorus with rising tones. The electron hybrid code simulations revealed that chorus with rising tones are generated when the wave amplitude of whistler-mode waves exceeds the threshold. The simulation results also revealed that the frequency profile of the wave amplitude of the reproduced chorus follows those of the theoretically estimated optimum wave amplitude. These results clarify that the generation process of chorus with rising tones is consistent with the nonlinear wave growth theory (Omura et al. 2008, 2009). The electron hybrid code simulations suggested that the threshold amplitude for the chorus generation varies depending on the background magnetic field inhomogeneity; a small magnetic field inhomogeneity along a field line lowers the threshold, allowing the triggering process of rising tone chorus to emerge easily in the equatorial region of the magnetosphere (Katoh and Omura 2013). The generation process of chorus emissions has also been reproduced in full particle code simulations by Hikishima et al. (2009). Hikishima et al. (2010) revealed that microburst precipitation of energetic electrons should occur due to the pitch angle scattering by the generated chorus emissions with rising tones. With high computational costs such as 256 cores for 2 days of a supercomputer, the electron hybrid and full particle codes enable us to carry out self-consistent wave-particle interactions in the magnetosphere.

While the previous simulation studies by the electron hybrid code used a spatially downscaled simulation system with a steep gradient of the background magnetic field intensity, recent computational resources enable us to use realistic conditions for the background magnetic field and the initial velocity distribution of energetic electrons (Katoh and Omura 2016). A spatially twodimensional code has also been developed for the study of the propagation properties of chorus emissions in the inner magnetosphere (Katoh 2014). Simulation studies of the interaction between chorus emissions and energetic 
electrons in comparison with in situ observation of the Arase satellite in the magnetosphere are important research subjects in future studies.

\section{Ion hybrid code}

Electromagnetic ion cyclotron (EMIC) wave, which is also an important target for Arase satellite, is generated by proton temperature anisotropy in the magnetosphere (e.g., Kennel and Petschek 1966). The nonlinear wave growth of the EMIC waves with rising frequency has been studied by theories (Omura et al. 2010), simulations (Shoji and Omura 2011, 2012, 2013, 2014), and observations (Pickett et al. 2010; Nakamura et al. 2014, 2015). In order to solve the ion scale dynamics with realistic parameters, we use the self-consistent ion hybrid simulations. The ion hybrid simulation treats the ions as particles and the electrons as mass-less fluid. Basic equations of the hybrid simulation are given as

$$
\begin{aligned}
& \nabla \times \boldsymbol{E}=-\frac{\partial \boldsymbol{B}}{\partial t} \\
& \nabla \times \boldsymbol{B}=\mu_{0} \boldsymbol{J} \\
& -\mathrm{en}_{\mathrm{e}} \boldsymbol{E}+\boldsymbol{J}_{\mathrm{e}} \times \boldsymbol{B}-\nabla p_{\mathrm{e}}=0 \\
& \frac{\mathrm{d} \boldsymbol{v}_{\mathrm{s}}}{\mathrm{d} t}=\frac{q_{\mathrm{s}}}{m_{\mathrm{s}}}\left(\boldsymbol{E}+\boldsymbol{v}_{\boldsymbol{s}} \times \boldsymbol{B}\right)
\end{aligned}
$$

where $E, B, J$, and $J_{\mathrm{e}}$ are the electric field, magnetic field, current density, and electron current density, respectively. $v_{\mathrm{s}}, q_{\mathrm{s}}$, and $m_{\mathrm{s}}$ are the velocity, charge density, and mass for particle species $s$, respectively.

Because the EMIC waves are generated in the parallel direction to the background magnetic field at the equator, the one-dimensional (1D) simulation model with open boundaries can be used for the reproduction of the nonlinear wave generation. We used a parabolic magnetic field to model the dipole-like magnetic field around the equatorial region as follows

$$
B_{0 x}=B_{0 \mathrm{eq}}\left(1+a x^{2}\right)
$$

where $B_{0 \mathrm{eq}}$ is the equatorial magnetic field strength, $x$ is the distance from the equator, $a$ is the magnetic field gradient $a=4.5 /\left(L R_{\mathrm{E}}\right)^{2}, L$ is the $L$ value, and $R_{\mathrm{E}}$ is the Earth's radius. To satisfy the condition $\nabla \cdot \boldsymbol{B}=0$, the perpendicular component of the magnetic field $\mathrm{B}_{\perp}$ is calculated as $B_{\perp}=-\frac{r_{L}}{2} \frac{\partial B_{x}}{\partial x}$, where $r_{\mathrm{L}}$ is the Larmor radius.

The particle distributions of the energetic protons, which are the source of the EMIC waves, is assumed as a subtracted Maxwellian, which is a distribution function modeling the loss cone distribution (Baumjohann and Treumann 1997).
For the realistic model, three components of ions cold $\mathrm{H}+, \mathrm{He}+$, and $\mathrm{O}+$ are uniformly distributed in the simulation space. In the present study, the external current source to excite monochromatic EMIC wave at $1.5 \mathrm{~Hz}$ is used. In the model, the rising tone EMIC waves are triggered by the monochromatic wave by consuming the free energy of the anisotropic protons. Here, the same initial amplitude and a different hot proton density are assumed in runs b, c, and d as shown in Table 1. As the hot proton density becomes larger, the frequency sweep rate becomes higher and the EMIC wave obtains larger wave amplitude as shown in panels b-d.

Figure 10 shows the dynamic spectra of the EMIC rising tone emissions with different initial wave amplitudes and energetic proton densities. Table 1 shows the detailed parameters for each panel. For other parameters, we used the realistic parameters observed by Cluster, as shown in Shoji and Omura (2011). In panel a, the EMIC wave is not excited since the distribution function of the energetic proton itself is stable. With small amplitude injections, we find rising tones in panels b, c, d, and e. With the same initial amplitude, shown in panels b, c, and d, the rising frequency becomes higher and the wave amplitude becomes larger as the initial density becomes larger. This is because the rising tone emissions for each parameter have different optimum wave growth condition. In panel e for the run with the highest density ratio, two rising tone elements appear. In this case, the first rising tone emission does not consume all free energy so a second one is triggered. In panel $\mathrm{f}$, with the strongest initial wave amplitude, the initial wave cannot trigger the rising tone emissions. Since the initial condition does not satisfy the optimum conditions, the nonlinear wave growth do not take place.

\section{A global ionospheric potential solver: GEMSIS-POT}

In order to implement an inner boundary condition for the above-described global models and to understand effects of the magnetosphere originated disturbances

Table 1 Initial parameters for hot plasma density normalized by the cold proton density and initial triggering wave amplitude

\begin{tabular}{lll}
\hline Run & $\begin{array}{l}\text { Hot proton } \\
\text { density }(\mathbf{n H})\end{array}$ & $\begin{array}{l}\text { Initial wave } \\
\text { amplitude (nT) }\end{array}$ \\
\hline Run (a) & 0.05 & 0. \\
Run (b) & 0.01 & 0.50 \\
Run (c) & 0.025 & 0.50 \\
Run (d) & 0.05 & 0.50 \\
Run (e) & 0.1 & 0.25 \\
Run (f) & 0.05 & 5.00 \\
\hline
\end{tabular}



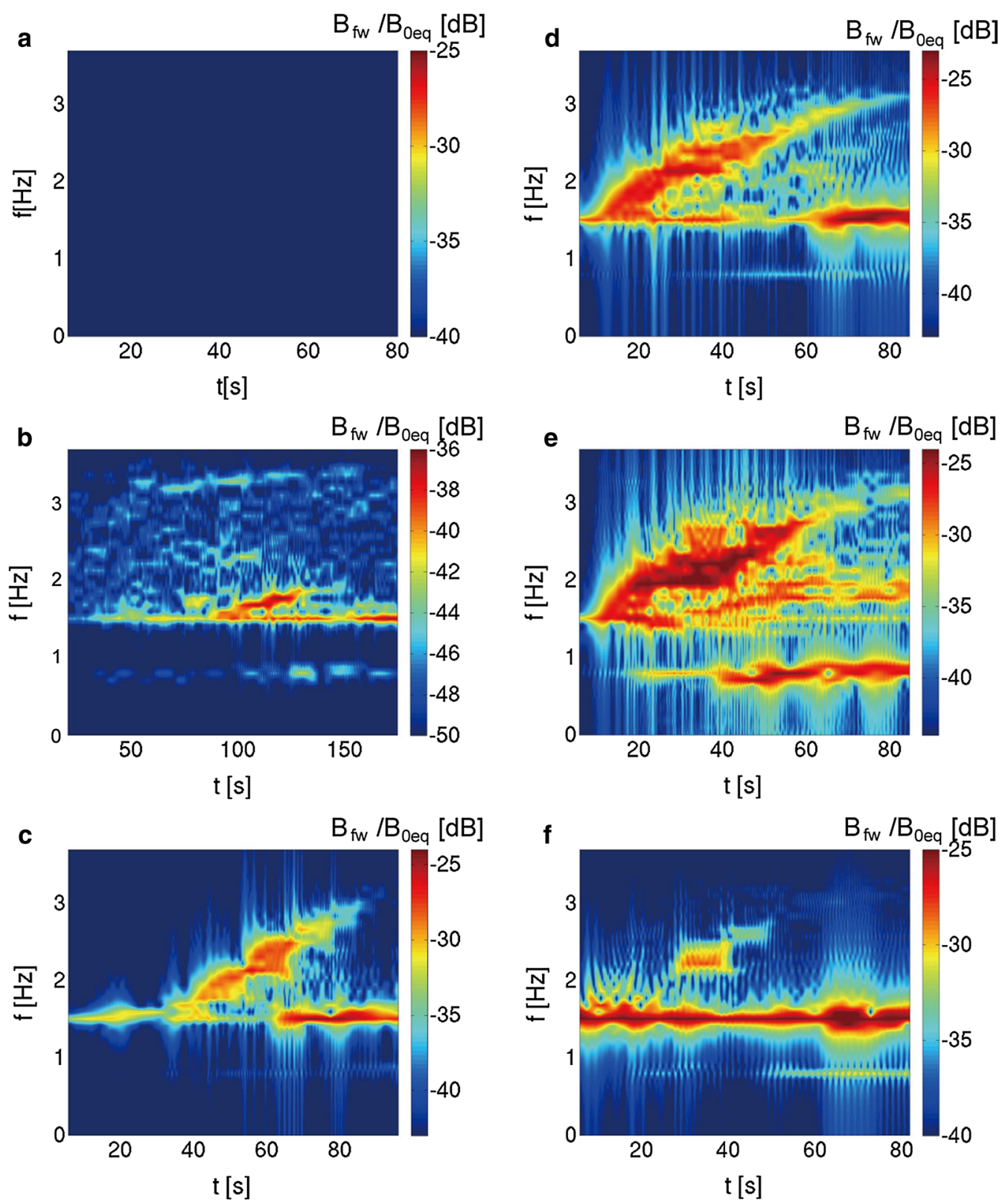

Fig. 10 Dynamic spectra of forward propagating waves with different parameters described in Table 1

on the global ionospheric electric field and current system, we developed a global ionospheric potential solver, which we call the GEMSIS-POT solver (Geospace Environment Modeling System for Integrated StudiesPOTential solver) (Nakamizo et al. 2012). GEMSIS-POT is basically the same as the so-called thin shell model, the concept of which has been used in many studies for more than half a century to derive the ionospheric electric field and current distributions (e.g., Fejer 1953; Nopper and Carovillano 1978; Kamide and Matsushita
1979; Harel et al. 1981; Senior and Blanc 1984; Tsunomura and Araki 1984). The formulation is exactly the same form as described by Amm (1996). This type of the solver has also been used as an inner boundary condition in global MHD models (e.g., Tanaka 1995; Janhunen 1998; Gombosi et al. 2000). One of the two extensions of GEMSIS-POT from the previous studies is that it is applied to the global ionosphere without placing any boundary at the equator, and the other important extension is a modification of conductance 
distribution in low-latitude region (described below) in relation to the former extension.

The procedure of the solver is as follows. We adopt a longitude $(\phi)$-latitude $(\theta)$ coordinate system assuming a sheet (2D) ionosphere. By relating the Ohm's law in the 2D ionosphere,

$$
\begin{aligned}
& \mathbf{J}=\boldsymbol{\Sigma} \cdot \mathbf{E} \\
& \mathbf{E}=-\nabla \Phi
\end{aligned}
$$

to the current continuity between the divergence of ionospheric currents and FACs flowing into/away from the ionosphere,

$$
-j_{\|} \sin I=-\nabla \cdot \mathbf{J}
$$

a Poisson equation for a conducting ionosphere is obtained:

$$
\begin{aligned}
& -j_{\|} \sin I=\nabla \cdot[\boldsymbol{\Sigma} \cdot \nabla \Phi] \\
& \boldsymbol{\Sigma}=\left(\begin{array}{cc}
\Sigma_{\theta \theta} & \Sigma_{\theta \varphi} \\
\Sigma_{-\theta \varphi} & \Sigma_{\theta \theta}
\end{array}\right),
\end{aligned}
$$

where $\mathbf{E}$ is the electric field, $\Phi$ is electric potential, $\mathbf{J}$ is ionospheric height-integrated current density, $j_{\|}$is current density of FACs (positive for downward), $\Sigma$ is heightintegrated conductance tensor, and $I$ is the dip angle of the local magnetic field. The solver, based on Eq. (5), gives $\Phi$ with $j_{\|}$as a source, which can be given observationally or by numerical models, under the prescribed $\boldsymbol{\Sigma}$ distribution.

GEMSIS-POT equips a conductance calculation tool, which gives a global conductance distribution in the following way. At first the values of $\sigma_{0}, \sigma_{\mathrm{P}}$, and $\sigma_{\mathrm{H}}$ at each point and height in the 3D ionosphere are calculated by using the neutral gas temperature and number densities of neutral species obtained from the NRLMSISE-00 model (Picone et al. 2002) and temperatures and number densities of charged particles obtained from the IRI2007 model (Bilitza and Reinisch 2008). Here we referred to Schunk and Walker (1973) and Schunk and Nagy (1978) for collision frequencies and referred to Brekke and Moen (1993) for the calculation formulae. As for the ambient magnetic field, the IGRF-2005 reference model or dipole field is used. By integrating the $\sigma_{0}, \sigma_{\mathrm{P}}$, and $\sigma_{\mathrm{H}}$ in the altitude from 90 to $300 \mathrm{~km}, \Sigma_{\theta \theta}, \Sigma_{\phi \phi}$, and $\Sigma_{\theta \phi}$ are obtained. During the course of the height integration, the modification for low-latitude region is performed (Tsunomura 1999; Nakamizo et al. 2012). Conductance enhancement associated with auroral activities can also be included.

Figure 11 shows a calculation example. Each panel adopts the same format with noon being ahead to the left and the north pole to the top. The calculation setting is as follows. The spatial resolution of the computational grid is set at $1.4^{\circ}$ for the $\phi$ direction and $0.7^{\circ}$ for the $\theta$ direction. The dipole field is used for the ambient magnetic field. The conductance distribution is calculated assuming an equinox condition with $a p=48$ (used in the IRI-2001 model). Conductance enhancements in auroral region is taken into account by referring Hardy et al. (1987) with $K p=5$. The FAC distribution is given by the following Gaussian function assuming a region 1 (R1)type current (Iijima and Potemra 1976)

$$
j_{\|}= \pm j_{0, R 1} \exp \left[-\frac{\left(\theta-\theta_{0, R 1}\right)^{2}}{\delta_{\theta, R 1}^{2}}-\frac{\left(\phi \mp \phi_{0, R 1}\right)^{2}}{\delta_{\phi, R 1}^{2}}\right],(7)
$$

where the subscripts "R1" denote R1-FAC, $j_{0, R 1}$ the peak current densities, $\theta_{0, R 1}$ the peak latitudes, $\phi_{0, R 1}$ the peak longitudes, $\delta_{\theta, R 1}$ the latitudinal e-folding distance, $\delta_{\phi, R 1}$ the longitudinal e-folding distance width, and the upper and lower signs are taken for the currents flowing into and away from the ionosphere, respectively. Specifically, $j_{0, R 1}=1.6 \mu \mathrm{A} / \mathrm{m}^{2}, \theta_{0, R 1}=70^{\circ}, \phi_{0, R 1}=90^{\circ}$ (equals to 06:00 and 18:00 in local time), $\delta_{\theta, R 1}=2^{\circ}$, and $\delta_{\phi, R 1}=45^{\circ}$ in this example. Figure 11a, b shows $\Sigma_{\theta \theta}$ in a logarithmic scale and input FAC in a linear scale by color, with positive value (red color) corresponding to the current into the ionosphere. Figure 11c shows the obtained ionospheric potential $\Phi$. We can see a familiar two-cell pattern of positive-negative peaks on the dawn and dusk hemispheres, respectively, corresponding to the input R1-FAC.

An advantage of the GEMSIS-POT model is its seamless coverage of the whole globe including the equatorial region. The seamless description of the ionospheric potential is important when we address inner magnetospheric phenomena such as the shielding effects due to the R2 current system and SAPS in the subauroral region, which affects the dynamics of the ring current ions and coupling between global inner magnetospheric simulations such as CIMI with REPPU ("Comprehensive Inner Magnetosphere-Ionosphere Model (CIMI) with global MHD simulation REPPU" section) and GEMSISRC ("Global drift-kinetic simulation of the ring current: GEMSIS-RC model" section). Comparison with Arase observations will allow us to investigate roles of M-I coupling in the inner magnetospheric dynamics.

\section{Empirical ionospheric electric field models based on SuperDARN observations and data assimilation}

Among the ground-based observations available to date, the Super Dual Auroral Radar Network (SuperDARN) (Greenwald et al. 1995) is one of the most powerful tools to diagnose ionospheric plasma convection of various scales (e.g., Chisham et al. 2007; Lester 2013). Although SuperDARN has the extensive field of view from the 


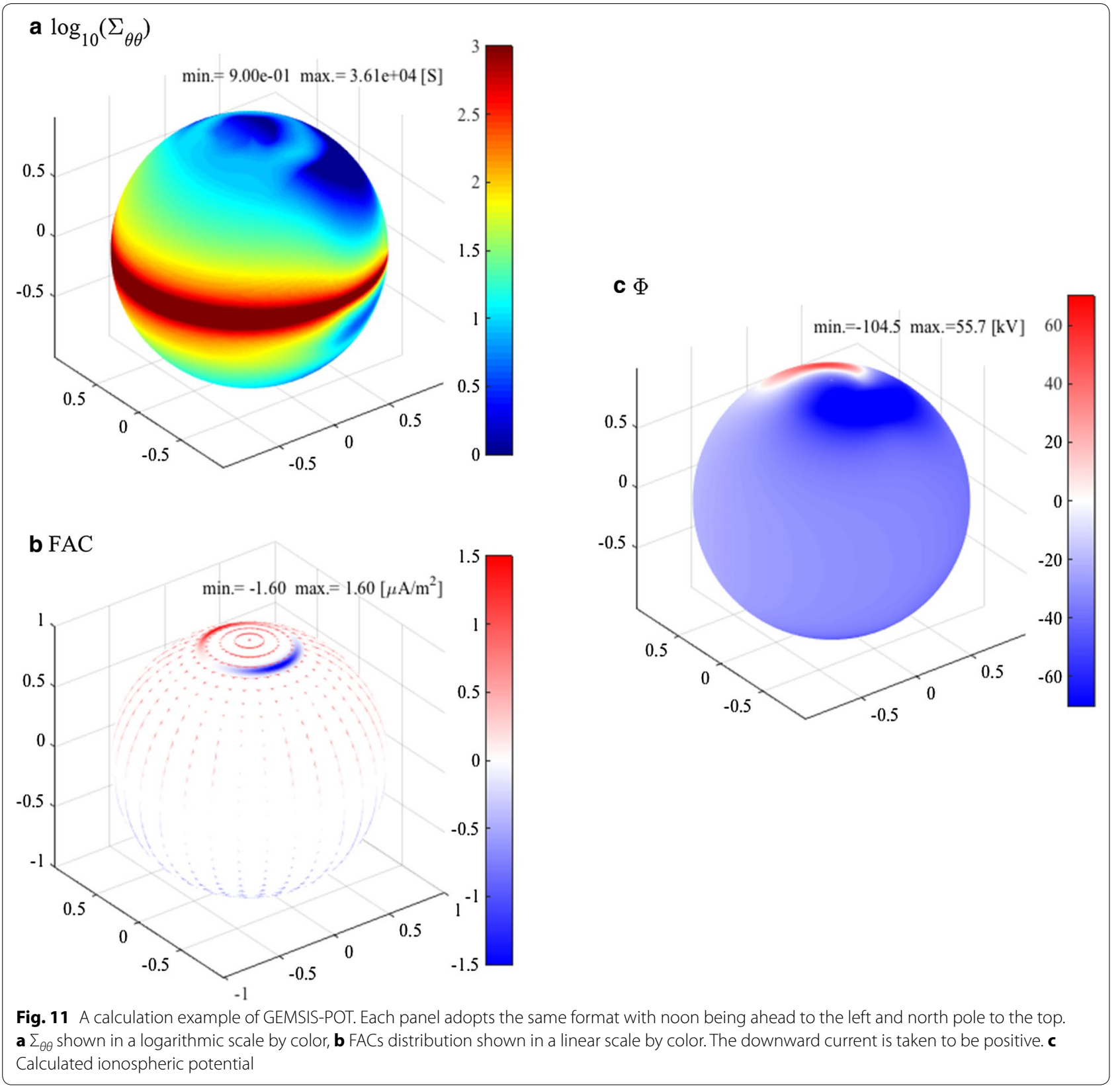

subauroral to polar region of both northern and southern hemispheres, the actual coverage of detected backscatter echoes can be sparse in the field of view of each radar. In addition to the echo coverage, a single radar provides us with only 1-dimensional (1-D) line-of-sight velocity (LOSV) vectors and we have to combine multiple LOSV vectors obtained in a common volume of the ionosphere by a pair of two neighboring radars to get a 2-dimensional (2-D) flow vector. To deduce a 2-D flow map by merging and spatially interpolating LOSV values, several modeling techniques based on the LOSV observation were developed in past studies. One of those methods, which is particularly suitable for estimating mesoscale ( a few thousand $\mathrm{km}$ ) structures of ionospheric convection, is the 2-D flow reconstruction with Spherical Elementary Current Systems (SECS) presented by Amm et al. (2010). In this method, observed LOSV vectors are fitted to a polynomial expansion of the SECS basis functions, which were originally introduced to express the ionospheric equivalent current based on geomagnetic field observations (Amm 1997; Amm and Viljanen 1999). An advantage comparing to the conventional map potential 
technique with spherical harmonic expansion (Ruohoniemi and Greenwald 1998) is that the SECS reconstruction method uses the non-periodic functions for the expansion of 2-D flow map and thus is not suffered from spurious flow structures generated by the periodicity of spherical harmonics.

An example of the estimate of ionospheric convection map with the SECS reconstruction technique is shown in Fig. 12. The left panel of the figure shows line-of-sight velocity (LOSV) vectors measured by the Christmas Valley East (CVE) radar and the Fort Hays West (FHW) radar, both of which are located in the mid-latitude region of North America, for 0930:00-0931:45 UT on March 17, 2015. The same LOSV values are also shown on the right panel but with color-coded pixels. Blue crosses gridded over the analyzed area usually referred to as "SECS poles" are where SECS basis functions are located. Black pins superposed on the right panel denote the estimated horizontal flow map resulting from the SECS reconstruction. The detailed analysis of the estimated convection map for this particular event has been given in a separate article.

The resultant velocity vectors show that ionospheric convection flows mainly eastward with speeds of several hundred $\mathrm{m} / \mathrm{s}$ in the region of magnetic latitudes of $\sim 50-60^{\circ}$. Comparison with the equi-latitude curves of the magnetic coordinates indicates that the flow is roughly aligned with the magnetic longitude direction rather than the geographical longitude direction. These flow properties are basically consistent with the enhanced sunward convection in the subauroral region on the dawnside during geomagnetically active times (e.g., Baker et al. 2007). Note that the resultant velocity estimates are obtained as 2-D horizontal velocity vectors from the present technique; the method provides a way to overcome limitations and caveats arising from the essentially 1-D velocity measurement of SuperDARN (e.g., Ponomarenko et al. 2001).

While Fig. 12 shows a regional structure of the ionospheric flow, it is also essential to know the global electric field distribution in studies of the physical processes in the inner magnetosphere which are to be conducted through the Arase project. Nakano et al. $(2008,2014)$ had conducted data assimilation of remote imaging measurements from the IMAGE satellite into a ring current model and a plasmasphere model, each of which forms a part of the CIMI model described in "Comprehensive Inner Magnetosphere-Ionosphere Model (CIMI) with global MHD simulation REPPU" section. They demonstrated that the global electric field structure is a key factor for modeling the temporal evolution of the inner magnetospheric state. The SuperDARN, which covers a wide spatial range of the high-latitude ionosphere, would be a powerful tool for monitoring the global electric field distribution. However, there are some wide gaps in the spatial coverage of the SuperDARN. In addition, as described above, each radar gives only the line-of-sight component of drift velocity and the data are frequently missing. Thus, it is not a trivial task to retrieve a global map of plasma drift velocity distribution from the SuperDARN data. Ruohoniemi and Baker (1998) developed a method to estimate a global map of drift velocity distribution from the SuperDARN data using the spherical harmonic fitting. Cousins et al. (2013) used empirical orthogonal functions as basis functions. These methods

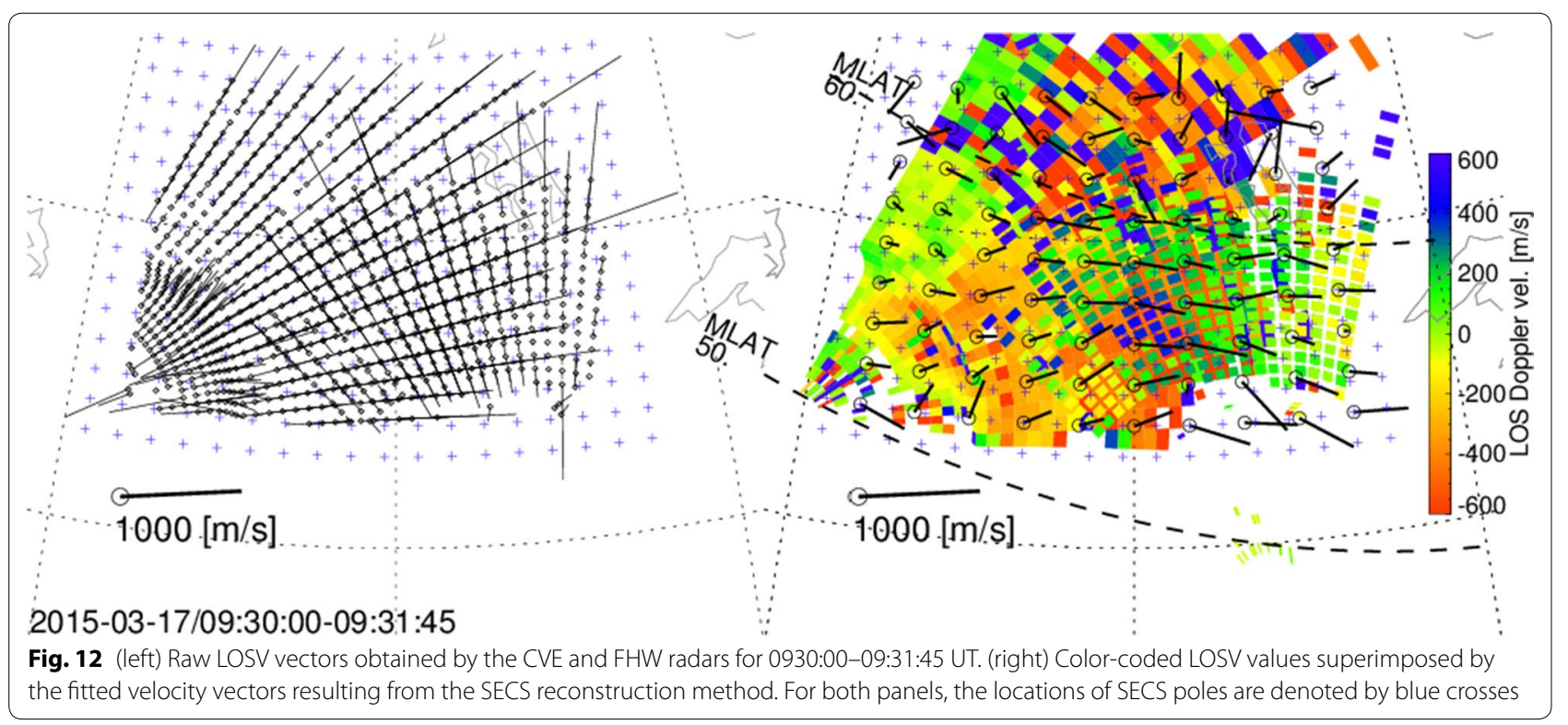


reduce the degree of freedom in order to reasonably estimate the global distribution. However, mesoscale structures which the SuperDARN can resolve are ignored.

We are developing a new method based on a different approach. If plasma drift velocity is assumed to be divergence-free, we can consider a stream function yielding the plasma velocity distribution. We express the stream function by a linear combination of kernel functions and estimate this stream function from the SuperDARN data. Since each kernel function represents a local structure in the vicinity of the center of the kernel function, the local structure as small as the width of the kernel function can be estimated for the region covered by the SuperDARN. In our proposed method, an empirical model can be combined into the estimate of the plasma velocity distribution. Thus, the gaps in the spatial coverage of the SuperDARN can be filled with the empirical model. The use of kernel functions representing a local structure is similar to the approach of Amm (1998) who used different kernel functions.

Figure 13 demonstrates an example of estimation results of our proposed method at 10:20 UT on March 27, 2017, when a small magnetic storm was developing. According to OMNI solar wind data, the interplanetary magnetic field (IMF) was southward from 09:00 UT. The IMF turned northward at 10:15 UT but the dawnward component (-By) did not decay. Hence, the ionospheric convection was supposed to be maintained. The AL index rapidly decreased around 10:00 UT, and it was around - $1200 \mathrm{nT}$ at 10:20 UT, which suggested that a substorm was underway. The left panel shows the estimated stream function. In the right panel, the estimated drift velocity distribution is shown with white arrows and the distribution of the uncertainty of the drift velocity is shown with color scale. When obtaining the result shown in this figure, the Weimer 2000 model (Weimer 2000) was combined into the estimate, which means the Weimer 2000 model is used for guessing the drift velocity in the regions where the SuperDARN data are not available. However, in such regions, the uncertainty is evaluated to be large. The Weimer model predicted a broad eastward drift region around 2-6 MLT. However, if the SuperDARN data were combined into the model, the eastward drift region was estimated to be confined within 30-35 degrees in colatitude (55-60 degree in latitude). During magnetic storms and substorms, meso- and large-scale convection patterns can vary significantly. The proposed method aims at obtaining a dynamical picture of variations of convection which could not be obtained by existing empirical models in order to reinforce the Arase and PWING (Shiokawa et al. 2017) observations.

\section{ERG (Arase) science center tools}

ERG (Arase) science center has developed data analysis tools for Arase data as well as for those from other spacecraft missions and ground-based observation networks. The tools facilitate integrated data analysis which combines different types/sources of observational data and modeling data. ERG science center makes the tools open to the public through websites and software packages. In this subsection, we introduce some important features of the tools, particularly in terms of simultaneous/conjugate observations between spacecraft and ground-based observations, quick plotting on the web, seamless data access and analysis, and calculation and visualization of plasma characteristics and wave properties.
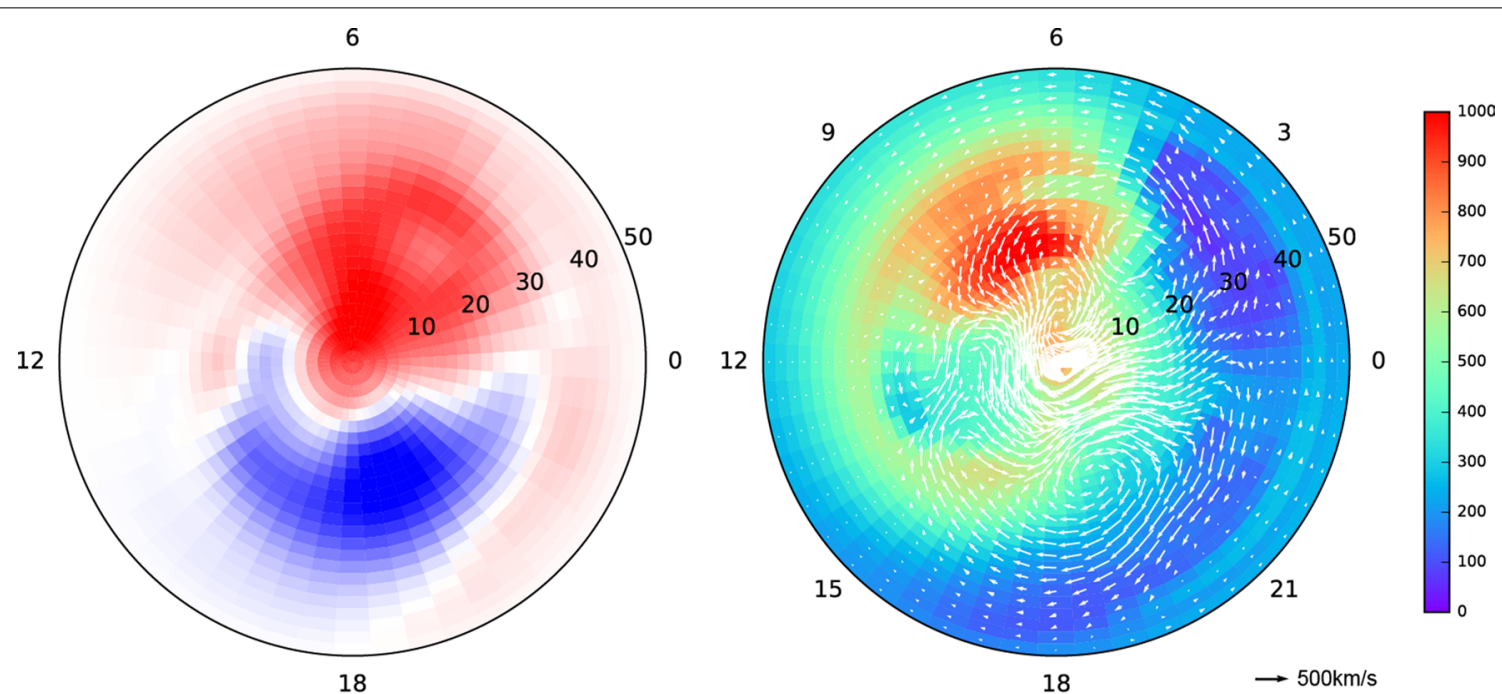

Fig. 13 The estimated stream function (left) and drift velocity distribution (right) on March 27, 2017, at 10:20 UT. In the right left panel, the estimated drift velocity distribution is shown with white arrows and the uncertainty of the drift velocity is shown with color scale 
Conjunction Event Finder (CEF at http://ergsc.isee. nagoya-u.ac.jp/cef/orbit.cgi) is a web-based graphical user interface that provides two useful functions: global maps for conjunctions between spacecraft and ground-based observations, and access to quick-look (QL) plots from a large number of different kinds of spacecraft and groundbased instrument in geospace physics. The global maps include a map on the northern (southern) hemisphere centered at the magnetic north (south) pole along with spacecraft orbit projected onto the $X-Y$ and $X-Z$ planes in GSM coordinates (Fig. 14). The map displays spacecraft footprints, locations of geomagnetic observatories, and field of views of all-sky cameras and radars. Once users select a date, CEF returns a list of links to QL plots that are provided by the mission PI teams. See details of all functions in the letter by Miyashita et al. (2011).

ERG Web Analysis Tool (ERGWAT at http://ergsc.isee. nagoya-u.ac.jp/analysis/ergwat/) provides a web-based,

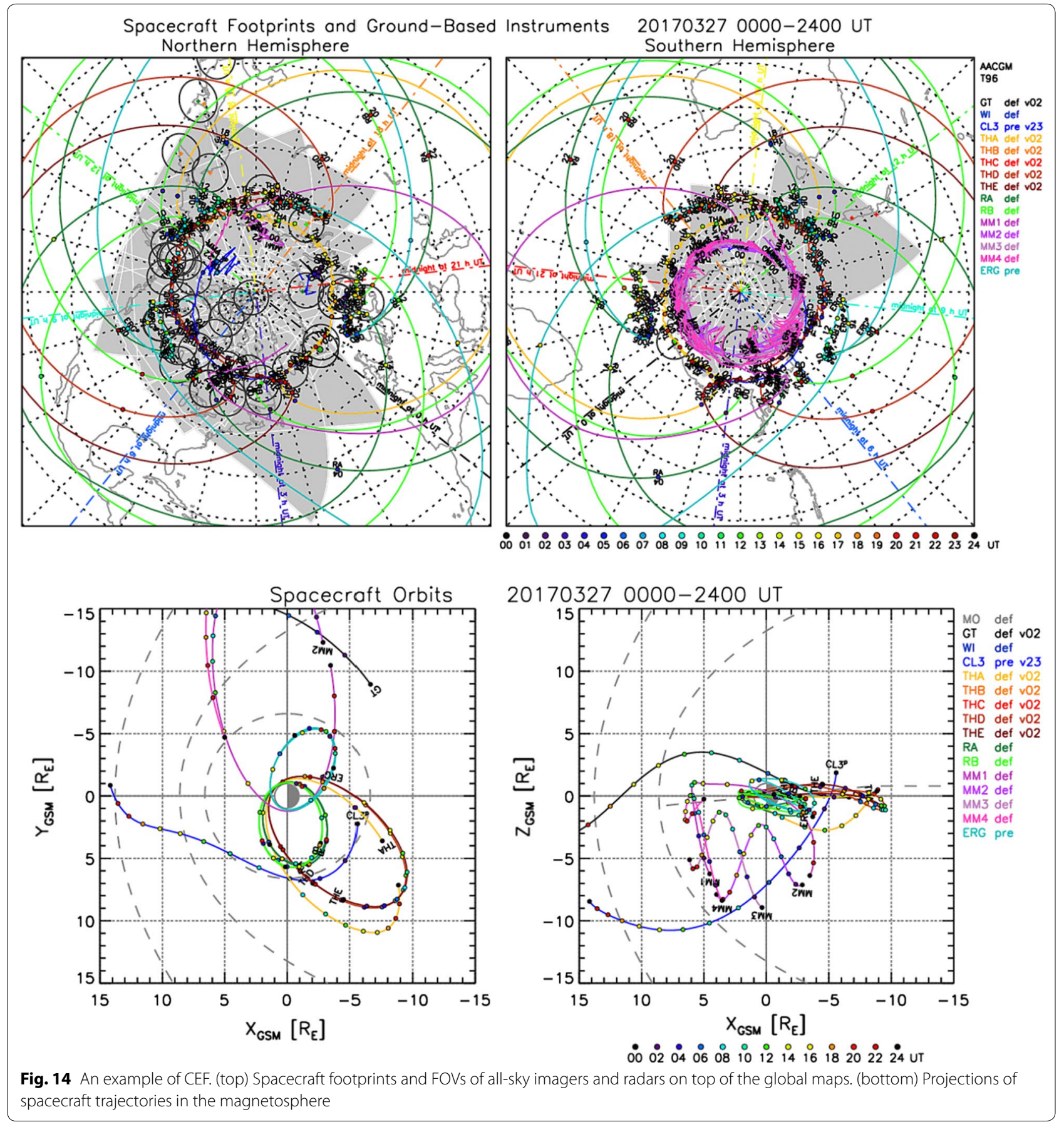


interactive tool to visualize and analyze various kinds of scientific data from geospace spacecraft and groundbased observations. The tool utilizes Space Physics Environmental Data Analysis Software (SPEDAS), which is written in Interactive Data Language (IDL), a commercial software for data manipulation and visualization, to load data via Internet and displays plots on a web browser. Plots can be stored in users' local computers as postscript files as well as image files for publications. System structure and sequence diagrams are detailed by Umemura et al. (2016).

ERG science center has also developed a large number of program codes to download and analyze spacecraft and ground-based observation data. The codes have been made available to international science community at https://ergsc.isee.nagoya-u.ac.jp/analysis/spedas/index. shtml.ja\#ERGplug-in, which is also released as a package of plug-in software libraries for SPEDAS. The package provides data users with seamless access to data (downloading via Internet, loading manipulating on local computers, visualizing, analyzing, etc.) across various kinds of projects and missions. The codes are compatible with data files in common data format (CDF). Plug-ins for groundbased observation data are detailed by Hori et al. (2015).

Useful tools about plasma and wave data are ISEE_3D and Kyoto University Plasma Dispersion Analysis Package (KUPDAP). The former is an interactive visualization tool of three-dimensional plasma velocity distribution function that provides a variety of ways to visualize the distribution function of space plasma. ISEE_3D also has a wide range of functions such as display of magnetic field vectors and two-dimensional slice of distributions. The source codes are written as scripts of IDL and made available as a plug-in of SPEDAS. Details of functions and examples are summarized by Keika et al. (2017). KUPDAP is a full dispersion solver (Sugiyama et al. 2015) developed by the space group at RISH, Kyoto University (http:// space.rish.kyoto-u.ac.jp/software/). The IDL codes and dynamic link modules (DLMs) are available as an ERG plug-in tool, implemented by ERG science center (https:// ergsc.isee.nagoya-u.ac.jp/analysis/spedas/kupdap/index. shtml). An interface between KUPDAP and observed plasma data is also available in the ERG plug-in package.

\section{Discussions on roles of integrated studies}

In this section, we will discuss the role of integrated studies. The approach incorporates the numerical models described in "Simulation and empirical models related to the ERG project" section with various types of observations including both the satellite and ground-based observations in order to understand dynamic variation in the geospace. To show a good example of the integrated approach, here we present investigation of internal acceleration process of the relativistic electrons in the outer radiation belt as one of the good examples of outstanding problems in the field.

Energization of relativistic electrons is a product of cross-energy couplings between charged particles within a wide energy range (from less than $\mathrm{eV}$ to hundreds of $\mathrm{keV}$ ) through the time evolution of electromagnetic fields within a wide frequency range (from DC to VLF or higher), which are closely connected to each other in the inner magnetosphere. The theory, modeling, and integrated studies contribute to revealing the causal relationship by providing physical interpretations of elements as well as the connection between them. Results of the modeling studies provide the evolution of the electromagnetic fields and/or the dynamics of charged particles in an ideal situation but spatially dense dataset, which contribute to the understanding of physical pictures of in situ but spatially sparse satellite observation data.

Since each piece of the causal relationship evolves in a different timescale, it is essential to combine results of multiple modeling studies employing different physical approaches. A study of whistler-mode wave-particle interaction should be a good example showing the significance of the integrated studies. On the energization process of relativistic electrons, particle simulations (both self-consistent (e.g., Katoh and Omura 2007b; "Electron hybrid code" section) and test particle ("Relativistic guiding center test particle model: GEMSIS-RB model" section) simulations) revealed that whistler-mode chorus emissions play an essential role through nonlinear wave-particle interactions, which occur in the timescale less than a second. Self-consistent particle simulations described in "Electron hybrid code" section (e.g., Katoh and Omura 2007a; Hikishima et al. 2009) revealed that the condition required for the chorus generation is controlled by various factors, at least by the intensity of both flux and anisotropy of energetic electrons (Katoh and Omura 2011) and the inhomogeneity of the background magnetic field (Katoh and Omura 2013). In order to understand where, when, and how the condition required for the chorus generation is satisfied, global models such as CIMI with REPPU give important clues by providing both the spatial and temporal evolution of the cold and/ or energetic electron populations as shown in "Comprehensive Inner Magnetosphere-Ionosphere Model (CIMI) with global MHD simulation REPPU" section and the modification of the background electromagnetic fields (e.g., Ebihara et al. 2014; Fig. 4). Both plasmaspheric density distribution ("Plasmasphere thermosphere model (PTM)" section) and the ionospheric electric potential ("A global ionospheric potential solver: GEMSIS-POT" section) can affect the dynamics of the ring current, and combination of these models will improve our 
understanding of the geospace environment as a coupled system. Most of these results of the global models can be consulted with reference to the results of self-consistent simulations of the chorus generation for identifying the region favorable to the relativistic electron energization by chorus emissions.

The results of integrated studies can also be used in the operation planning of the Arase satellite, particularly for the observation planning of software-type wave-particle interaction analyzer (S-WPIA) on board the Arase satellite (Katoh et al. 2018a; Hikishima et al., submitted to Earth, Planets and Space). S-WPIA challenges the direct measurement of wave-particle interactions between chorus emissions and relativistic electrons in the inner magnetosphere. Even though dedicated courtesy of the satellite operation enables us to use a certain amount of telemetry budget as much as possible, the duration of the S-WPIA measurement is intermittent with short duration for every orbit, since the size of electromagnetic waveform and individual particle count data is large. If we can consult the results of the integrated studies at a timing of the operation planning of the Arase satellite, we can maximize the possibility to measure good events by devoting the limited observation time of S-WPIA to the region of interest where we can expect efficient waveparticle interactions.

As described in the overview paper by Miyoshi et al. (this issue), there are three major science questions to be addressed in the ERG (Arase) project:

1. Which of external source process and internal acceleration process is dominant for the large flux enhancement of relativistic electrons during geomagnetic storms?

2. How do wave-particle interactions cause accelerations of relativistic electrons?

3. What are the dominant loss processes for relativistic electrons?

We already discussed how the models and integrated studies with observations can contribute to investigations of the internal acceleration processes as well as roles of wave-particle interactions in causing accelerations of relativistic electrons in details above. Here we briefly also discuss other science targets.

As for the external source process of the relativistic electrons in the outer radiation belt, the ULF waves have been considered as the promising driver of the radial transport essential to the process. The radial diffusion model described in "Radial diffusion model of the radiation belt electrons" section can be used as a good reference of empirical contribution of the radial diffusion process in relativistic electron variations. As shown in "Global drift-kinetic simulation of the ring current: GEMSIS-RC Model" section, combination of GEMSISRC ("Global drift-kinetic simulation of the ring current: GEMSIS-RC Model" section) and GEMSIS-RB ("Relativistic guiding center test particle model: GEMSIS-RB model" section) models provide us a new tool to investigate the efficiency of radial transport with Pc5 ULF waves. For more realistic ULF distributions in the inner magnetosphere, a coupling study with CCMC BATSRUS global MHD simulations (Tóth et al. 2005) and GEMSIS$\mathrm{RC}$ is also underway. Comparison with in situ fields and electron observations is essential to assessing model results as well as for quantitative understanding.

In order to investigate the dominant loss processes for relativistic electrons, similar combination of the global models ("Comprehensive Inner Magnetosphere-Ionosphere Model (CIMI) with global MHD simulation REPPU" section) and kinetic simulations ("Electron hybrid code" section) is useful for understanding the loss process through interaction with waves such as EMIC. The global model together with ground-based observations related to the Arase (ERG) project (Shiokawa et al. 2017) provides spatial distribution of the wave-particle interaction regions. Comparison of kinetic simulation results with electron pitch angle observations by the Arase satellite will provide essential clues for the loss process. As for the magnetopause shadowing process, combination between empirical magnetic field model and GEMSIS-RB (e.g., Saito et al. 2010) provides a good tool to evaluate contribution of the process quantitatively.

\section{Summary}

A review of the technical aspects of the numerical simulations and models and integrated analysis tools related to the geospace exploration project, ERG, is provided in this paper. The importance of the integrated studies which incorporate the models with observations in the researches of geospace variations is also discussed. This technical report includes the following models and tools: The radial diffusion model ("Radial diffusion model of the radiation belt electrons" section), relativistic guiding center test particle model (GEMSIS-RB model, "Relativistic guiding center test particle model: GEMSIS-RB model" section), CIMI with global MHD simulation REPPU ("Comprehensive Inner Magnetosphere-Ionosphere Model (CIMI) with global MHD simulation REPPU" section), global drift-kinetic model for the ring current (GEMSIS-RC, "Global drift-kinetic simulation of the ring current: GEMSIS-RC Model" section), plasmasphere thermosphere model (PTM, "Plasmasphere thermosphere model (PTM)" section), wave-particle interaction module for GEMSIS-RC (GEMSIS-RBW model, "Wave-particle interaction module for GEMSIS-RB (GEMSIS-RBW 
model)" section), self-consistent wave-particle interaction simulations with the electron hybrid code ("Electron hybrid code" section) and ion hybrid code (Ion hybrid code section), global ionospheric potential solver (GEMSIS-POT model, "A global ionospheric potential solver: GEMSIS-POT" section), empirical ionospheric electric field models based on SuperDARN observations and data assimilation ("Empirical ionospheric electric field models based on SuperDARN observations and data assimilation" section), and ERG (Arase) science center tools for integrated studies ("ERG (Arase) science center tools" section).

In each subsection, the characteristics of the simulation/model/tool, such as the advantage and technical limitation, are described together with references and some examples of application. This paper thus provides a catalog of models and tools for future integrated studies of the dynamic geospace variations, such as geomagnetic storms, in cooperation with related satellite and groundbased observations.

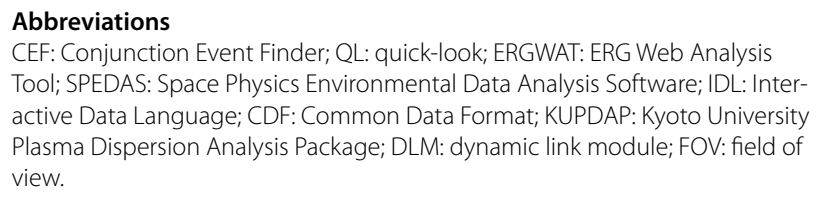

Abbreviations

CEF: Conjunction Event Finder; QL: quick-look; ERGWAT: ERG Web Analysis Tool; SPEDAS: Space Physics Environmental Data Analysis Software; IDL: Interactive Data Language; CDF: Common Data Format; KUPDAP: Kyoto University Plasma Dispersion Analysis Package; DLM: dynamic link module; FOV: field of view.

\section{Authors' contributions}

$\mathrm{KS}, \mathrm{YM}$, and NT carried out the planning and coordination of the paper together with contribution to the inner magnetospheric models. Authors mainly contributed to each subsection are YM ("Radial diffusion model of the radiation belt electrons" section), SS ("Relativistic guiding center test particle model: GEMSIS-RB model" and "Wave-particle interaction module for GEMSISRB (GEMSIS-RBW model)" sections), YE, MF, and TT ("Comprehensive Inner Magnetosphere-lonosphere Model (CIMI) with global MHD simulation REPPU" section), TA, K. Kamiya, and KS ("Global drift-kinetic simulation of the ring current: GEMSIS-RC Model" section), SW ("Plasmasphere Thermosphere Model (PTM)" section), YK and MS ("Self-consistent wave-particle interaction simulations" section), AN, AY, and Al ("A global ionospheric potential solver: GEMSISPOT" section), TH and NS ("Empirical ionospheric electric field models based on SuperDARN observations and data assimilation" section), and K. Keika ("ERG (Arase) science center tools" section), respectively. KS, YK, YM, YE, YO, and MN are main contributors to "Discussions on roles of integrated studies" section. All authors read and approved the final manuscript.

\section{Author details \\ ${ }^{1}$ Graduate School of Science, University of Tokyo, Tokyo, Japan. ${ }^{2}$ ISEE, Nagoya University, Nagoya, Japan. ${ }^{3}$ RISH, Kyoto University, Uji, Japan. ${ }^{4}$ Graduate School of Science, Tohoku University, Sendai, Japan. ${ }^{5}$ Graduate School of Sci- ence, Nagoya University, Nagoya, Japan. ${ }^{6}$ Applied Electromagnetic Research Institute, NICT, Tokyo, Japan. ${ }^{7}$ The Institute of Statistical Mathematics, Tokyo, Japan. ${ }^{8}$ Hokkaido Information University, Ebetsu, Japan. ${ }^{9}$ Graduate School of Science, Kyoto University, Kyoto, Japan. ${ }^{10}$ Goddard Space Flight Center, NASA, Greenbelt, MD, USA. ${ }^{11}$ ICSWSE, Kyushu University, Fukuoka, Japan.}

\section{Acknowledgements}

The ERG science center has been operated by ISAS/JAXA and ISEE/Nagoya University.

\section{Competing interests}

The authors declare that they have no competing interests.
Consent for publication

Not applicable.

Ethics approval and consent to participate

Not applicable.

Funding

This work was partially supported by JSPS KAKENHI Grant Numbers 16H06286, 16H02229, 15H05747, 15H05815, 15H03732, and 15H03730.

\section{Publisher's Note}

Springer Nature remains neutral with regard to jurisdictional claims in published maps and institutional affiliations.

Received: 15 September 2017 Accepted: 17 January 2018

Published online: 01 February 2018

\section{References}

Abe T, Whalen BA, Yau AW, Watanabe S, Sagawa E, Oyama K-I (1993) Altitude profile of the polar wind velocity and its relationship to ionospheric conditions. Geophys Res Lett 20:2825-2828

Abel B, Thorne RM (1998) Electron scattering loss in Earth's inner magnetosphere: 1. J Geophys Res 103:2385-2396

Albert JM (1999) Analysis of quasi-linear diffusion coefficients. J Geophys Res 104:2429-2441

Albert JM (2002) Nonlinear interaction of outer zone electrons with VLF waves. Geophys Res Lett 29(8):1275. https://doi.org/10.1029/2001GL013941

Amano T, Seki K, Miyoshi Y, Umeda T, Matsumoto Y, Ebihara Y, Saito S (2011) Self-consistent kinetic numerical simulation model for ring current particles in the Earth's inner magnetosphere. J Geophys Res 116:A02216. https://doi.org/10.1029/2010JA015682

Amm O (1996) Comment on "A three-dimensional, iterative mapping procedure for the implementation of an ionosphere-magnetosphere anisotropic Ohm's law boundary condition in global magnetohydrodynamic simulations" by Michael L. Goodman. Ann Geophys 14:773

Amm O (1997) lonospheric elementary current systems in spherical coordinates and their application. J Geomagn Geoelectr 49:947-955. https:// doi.org/10.5636/jgg.49.947

Amm O (1998) Method of characteristics in spherical geometry applied to a Harang discontinuity situation. Ann Geophys 16:413

Amm O, Viljanen A (1999) lonospheric disturbance magnetic field continuation from the ground to the ionosphere using spherical elementary current systems. Earth Planets Space 51:431-440. https://doi. org/10.1186/BF03352247

Amm O, Grocott A, Lester M, Yeoman TK (2010) lonospheric plasma convection from coherent scatter radar data using the SECS technique. J Geophys Res 115:A03304. https://doi.org/10.1029/2009ja014832

Bailey GA, Balan N, Su YZ (1997) The Sheffield University plasmasphere ionosphere model_a review. J Atmos Terr Phys 59:1541-1552

Baker DN, Kanekal SG, Li X, Monk SP, Goldstein J, Burch JL (2004) An extreme distortion of the Van Allen belt arising from the 'Halloween' solar storm in 2003. Nature 432:878-881

Baker JBH, Greenwald RA, Ruohoniemi JM, Oksavik K, Gjerloev JW, Paxton L, Hairston MR (2007) Observations of ionospheric convection from the Wallops SuperDARN radar at middle latitudes. J Geophys Res 112:A01303. https://doi.org/10.1029/2006JA011982

Baker DN et al (2013) A long-lived relativistic electron storage ring embedded within the Earth's outer Van Allen radiation zone. Science 340(6129):186-190. https://doi.org/10.1126/science.1233518

Baumjohann W, Treumann RA (1997) Basic space plasma physics. Imperial College Press, London

Bilitza D, Reinisch BW (2008) International reference ionosphere 2007: improvements and new parameters. Adv Space Res 42:599-609. https://doi.org/10.1016/j.asr.2007.07.048

Borovsky JE, Thomsen MF, Elphic RC (1998) The driving of the plasma sheet by the solar wind. J Geophys Res 103:17617-17639. https://doi. org/10.1029/97JA02986 
Bortnik J, Thorne RM, Inan US (2008) Nonlinear interaction of energetic electrons with large amplitude chorus. Geophys Res Lett 35:L21102. https:// doi.org/10.1029/2008GL035500

Brautigam DH, Albert JM (2000) Radial diffusion analysis of outer radiation belt electrons during the October 9, 1990, magnetic storm. J Geophys Res 105(A1):291-309. https://doi.org/10.1029/1999JA900344

Brekke A, Moen J (1993) Observations of high latitude ionospheric conductances. J Atmos Terr Phys 55(11/12):1493-1512. https://doi. org/10.1016/0021-9169(93)90126-J

Brizard AJ, Chan AA (1999) Nonlinear relativistic gyrokinetic Vlasov-Maxwell equations. Phys Plasmas 6:4548. https://doi.org/10.1063/1.873742

Carpenter DL, Anderson RR (1992) An ISEE/Whistler model of equatorial electron density in the magnetosphere. J Geophys Res 97:1097-1108

Chen Y, Reeves GD, Friedel RHW (2007) The energization of relativistic electrons in the outer Van Allen radiation belt. Nat Phys 3:614-617. https:// doi.org/10.1038/nphys655

Chisham G, Lester M, Milan SE et al (2007) A decade of the Super Dual Auroral Radar Network (SuperDARN): scientific achievements, new techniques and future directions. Surv Geophys 28:33-109. https://doi. org/10.1007/s10712-007-9017-8

Cousins EDP, Matsuo T, Richmond AD (2013) SuperDARN assimilative mapping. J Geophys Res 118:7954-7962. https://doi.org/10.1002/2013JA019321

Degeling AW, Ozeke LG, Rankin R, Mann IR, Kabin K (2008) Drift resonant generation of peaked relativistic electron distributions by Pc 5 ULF waves. J. Geophys. Res. 113:A02208. https://doi.org/10.1029/2007JA012411

De Zeeuw DL, Sazykin S, Wolf RA, Gombosi TI, Ridley AJ, Tóth G (2004) Coupling of a global MHD code and an inner magnetospheric model: initial results. J Geophys Res Space Phys 109(A12):A12219. https://doi. org/10.1029/2003JA010366

Ebihara Y, Ejiri M (2000) Simulation study on fundamental properties of the storm-time ring current. J Geophys Res 105(A7):15843-15859. https:// doi.org/10.1029/1999JA900493

Ebihara Y, Ejiri M (2003) Numerical simulation of the ring current: review. Space Sci Rev 105:377-452

Ebihara Y, Miyoshi Y (2011) Dynamic inner magnetosphere: a tutorial and recent advances. In: Liu W, Fujimoto M (eds) The dynamic magnetosphere. IAGA special Sopron book series. Springer, New York. https:// doi.org/10.1007/978-94-007-0501-2_9

Ebihara Y, Tanaka T (2015a) Substorm simulation: insight into the mechanisms of initial brightening. J Geophys Res Space Phys 120:7270-7288. https:// doi.org/10.1002/2015JA021516

Ebihara Y, Tanaka T (2015b) Substorm simulation: formation of westward traveling surge. J Geophys Res Space Phys 120:10466-10484. https:// doi.org/10.1002/2015JA021697

Ebihara Y, Tanaka T (2016) Substorm simulation: quiet and N-S arcs preceding auroral breakup. J Geophys Res 121:1201-1218. https://doi. org/10.1002/2015JA021831

Ebihara Y, Tanaka T, Kikuchi T (2014) Counter equatorial electrojet and overshielding after substorm onset: global MHD simulation study. J Geophys Res 119:7281-7296. https://doi.org/10.1002/2014JA020065

Elkington SR, Hudson MK, Chan AA (1999) Acceleration of relativistic electrons via drift-resonant interaction with toroidal-mode Pc-5 ULF oscillations. Geophys Res Lett 26:3273-3276. https://doi. org/10.1029/1999GL003659

Fejer JA (1953) Semidiurnal currents and electron drifts in the ionosphere. J Atmos Terr Phys 4:184-203. https://doi. org/10.1016/0021-9169(53)90054-3

Fok M-C, Horne RB, Meredith NP, Glauert SA (2008) Radiation belt environment model: application to space weather nowcasting. J Geophys Res 113:1-12. https://doi.org/10.1029/2007JA012558

Fok M-C, Buzulukova NY, Chen S-H et al (2014) The Comprehensive Inner Magnetosphere-lonosphere Model. J Geophys Res Space Phys 119:75227540. https://doi.org/10.1002/2014JA020239

Friedel RHW, Reeves GD, Obara T (2002) Relativistic electron dynamics in the inner magnetosphere-a review. J Atmos Sol Terr Phys 64:265-282

Gaffey JD, Bilitza D (1994) NASA/National Space Science Data Center trapped radiation models. J Spacecr Rockets 31:172-176

Gallagher DL, Craven PD, Comfort RH (2000) Global core plasma model. J Geophys Res 105(A8):18819-18833

Gkioulidou M, Wang C-P, Lyons LR (2011) Effect of self-consistent magnetic field on plasma sheet penetration to the inner magnetosphere: rice convection model simulations combined with modified Dungey forcebalanced magnetic field solver. J Geophys Res 116:A12213. https://doi. org/10.1029/2011JA016810

Glocer A, Fok M, Meng X et al (2013) CRCM + BATS-R-US two-way coupling. J Geophys Res Space Phys 118:1635-1650. https://doi.org/10.1002/ jgra.50221

Gombosi TI, DeZeeuw DL, Groth CPT, Powell KG (2000) Magnetospheric configuration for Parker-spiral IMF conditions: results of a 3D AMR MHD simulation. Adv Space Res 26:139-149

Greenwald RA, Baker KB, Dudeney JR et al (1995) DARN/SuperDARN. Space Sci Rev 71:761-796. https://doi.org/10.1007/BF00751350

Hardy DA, Gussenhoven MS, Raistrick R, McNeil WJ (1987) Statistical and functional representations of the pattern of auroral energy flux, number flux, and conductivity. J Geophys Res 92(A11):12275-12294. https://doi. org/10.1029/JA092iA11p12275

Harel M, Wolf RA, Reiff PH, Spiro RW, Burke WJ, Rich FJ, Smiddy M (1981) Quantitative simulation of a magnetospheric substorm 1. Model logic and overview. J Geophys Res 86(A4):2217-2241. https://doi.org/10.1029/ JA086iA04p02217

Hedin AE (1991) Extension of the MSIS thermospheric model into the middle and lower atmosphere. J Geophys Res 96(A2):1159-1172. https://doi. org/10.1029/90JA02125

Hikishima M, Yagitani S, Omura Y, Nagano I (2009) Full particle simulation of whistler-mode rising chorus emissions in the magnetosphere. J Geophys Res Space Phys 114:A01203. https://doi. org/10.1029/2008JA013625

Hikishima M, Omura Y, Summers D (2010) Microburst precipitation of energetic electrons associated with chorus wave generation. Geophys Res Lett 37:L07103. https://doi.org/10.1029/2010GL042678

Hori T, Miyashita Y, Miyoshi Y, Keika K, Shoji M, Shinohara I, Shiokawa K, Otsuka Y, Abe S, Yoshikawa A, Yumoto K, Obana Y, Nishitani N, Yukimatu AS, Nagatsuma T, Kunitake M, Hosokawa K, Ogawa Y, Murata KT, Nose M, Kawano H, Sakanoi T (2015) CDF data archive and integrated data analysis platform for ERG-related ground data developed by ERG Science Center (ERG-SC). J Space Sci Info Jpn 4:75-89. JAXA-RR-14-009 (ISSN 1349-1113)

Huang X, Reinisch BW, Song P, Green JL, Gallagher DL (2004) Developing an empirical density model of the plasmasphere using IMAGE/RPI observations. Adv Space Res 33:829-832

lijima T, Potemra TA (1976) The amplitude distribution of field-aligned currents at northern high latitudes observed by Triad. J Geophys Res 81(13):2165-2174. https://doi.org/10.1029/JA081i013p02165

Janhunen P (1998) On the possibility of using an electromagnetic ionosphere in global MHD simulations. Ann Geophys 16:397-402. https://doi. org/10.1007/s005850050601

Jordanova VK, Zaharia S, Welling DT (2010) Comparative study of ring current development using empirical, dipolar, and self-consistent magnetic field simulations. J Geophys Res 115:A00J11. https://doi. org/10.1029/2010JA015671

Jordanova VK, Welling DT, Zaharia SG, Chen L, Thorne RM (2012) Modeling ring current ion and electron dynamics and plasma instabilities during a high-speed stream driven storm. J Geophys Res 117:A00L08. https://doi. org/10.1029/2011JA017433

Kamide Y, Matsushita S (1979) Simulation studies of ionospheric electric fields and currents in relation to field-aligned currents, 1. Quiet periods. J Geophys Res 84(A8):4083-4098. https://doi.org/10.1029/ JA084iA08p04083

Kamide Y, Baumjohann W, Daglis IA, Gonzalez WD, Grandes M, Joselyn JA, McPherron RL, Phillips JL, Reeves EGD, Rostoker G, Sharma AS, Singer HJ, Tsurutani BT, Vasyliunas VM (1998) Current understanding of magnetic storms: storm-substorm relationships. J Geophys Res 103(A8):17705-17728

Kasaba Y et al (2017) Wire Probe Antenna (WPT) and Electric Field Detector (EFD) of Plasma Wave Experiment (PWE) aboard the Arase satellite: specifications and initial evaluation results. Earth Planets Space 69:174. https://doi.org/10.1186/s40623-017-0760-x

Katoh Y (2014) A simulation study of the propagation of whistler-mode chorus in the Earth's inner magnetosphere. Earth Planets Space 66:6. https:// doi.org/10.1186/1880-5981-66-6

Katoh Y, Omura Y (2004) Acceleration of relativistic electrons due to resonant scattering by whistler mode waves generated by temperature 
anisotropy in the inner magnetosphere. J Geophys Res 109:A12214. https://doi.org/10.1029/2004JA010654

Katoh Y, Omura Y (2007a) Computer simulation of chorus wave generation in the Earth's inner magnetosphere. Geophys Res Lett 34:L03102. https:// doi.org/10.1029/2006GL028594

Katoh Y, Omura Y (2007b) Relativistic particle acceleration in the process of whistler-mode chorus wave generation. Geophys Res Lett 34:L029758. https://doi.org/10.1029/2007GL029758

Katoh Y, Omura Y (2011) Amplitude dependence of frequency sweep rates of whistler mode chorus emissions. J Geophys Res 116:A07201. https:// doi.org/10.1029/2011JA016496

Katoh Y, Omura Y (2013) Effect of the background magnetic field inhomogeneity on generation processes of whistler-mode chorus and broadband hiss-like emissions. J Geophys Res Space Phys 118:4189-4198. https:// doi.org/10.1002/jgra.50395

Katoh Y, Omura Y (2016) Electron hybrid code simulation of whistler-mode chorus generation with real parameters in the Earth's inner magnetosphere. Earth Planets Space 68:192. https://doi.org/10.1186/ s40623-016-0568-0

Katoh Y, Kojima H, Hikishima M, Takashima T, Asamura K, Miyoshi Y, Kasahara Y, Kasahara S, Mitani T, Higashio N, Matsuoka A, Ozaki M, Yagitani S, Yokota S, Matsuda S, Kitahara M, Shinohara I (2018a) Software-type WaveParticle Interaction Analyzer on board the Arase satellite. Earth Planets Space 70:4. https://doi.org/10.1186/s40623-017-0771-7

Katoh Y, Omura Y, Miyake Y, Usui H, Nakashima H (2018b) Dependence of generation of whistlermode chorus emissions on the temperature anisotropy and density of energetic electrons in the Earth's inner magnetosphere. J Geophys Res Space Phys 123. https://doi. org/10.1002/2017JA024801

Keika K, Miyoshi Y, Machida S, leda A, Seki K, Hori T, Miyashita Y, Shoji M, Shinohara I, Angelopoulos I, Lewis JW, Flores A (2017) A visualization tool for three-dimensional plasma velocity distributions (ISEE_3D) as a plug-in tool for SPEDAS. Earth Planets Space 69:170. https://doi.org/10.1186/ s40623-017-0761-9

Kellerman AC, Shprits YY, Kondrashov D, Subbotin D, Makarevich RA, Donovan E, Nagai T (2014) Three-dimensional data assimilation and reanalysis of radiation belt electrons: observations of a four-zone structure using five spacecraft and the VERB code. J Geophys Res Space Phys 119:87648783. https://doi.org/10.1002/2014JA020171

Kennel CF, Petschek HE (1966) Limit on stably trapped particle fluxes. J Geophys Res 71:1-28. https://doi.org/10.1029/JZ071i001p00001

Kim KC, Lee D-Y, Kim H-J, Lyons LR, Lee ES, Öztürk MK, Choi CR (2008) Numerical calculations of relativistic electron drift loss effect. J Geophys Res 113:A09212. https://doi.org/10.1029/2007JA013011

Koller J, Chen Y, Reeves GD, Friedel RHW, Cayton TE, Vrugt JA (2007) Identifying the radiation belt source region by data assimilation. J Geophys Res 112:A06244. https://doi.org/10.1029/2006JA012196

Kutiev I, Oyama K-I, Watanabe S, Abe T, Kumamoto A (2004) Plasmasphere electron temperature structures. Adv Space Res 34(9):2010-2015

Lester M (2013) The Super Dual Auroral Radar Network (SuperDARN): an overview of its development and science. Adv Polar Sci 24:1-11. https://doi. org/10.3724/SP.J.1085.2013.00001

Li X, Temerin M, Baker DN, Reeves GD, Larson D (2001) Quantitative prediction of radiation belt electrons at geostationary orbit based on solar wind measurements. Geophys Res Lett 28:1887-1890

Lorenzten KR, Looper MD, Blake JK (2001) Relativistic electron microbursts during the GEM storms. Geophys Res Lett 28:2573-2576. https://doi. org/10.1029/2001GL012926

Lyons LR, Thorne RM, Kennel CF (1972) Pitch-angle diffusion of radiation belt electrons within the plasmasphere. J Geophys Res 77:3455-3474

Maget V, Bourdarie Boscher SD, Friedel RHW (2007) Data assimilation of LANL satellite data into the Salammbô electron code over a complete solar cycle by direct insertion. Space Weather 5:S10003. https://doi. org/10.1029/2007SW000322

Meredith N, Horne R, Thorne R, Anderson R (2003) Favored regions for chorus-driven electron acceleration to relativistic energies in the Earth's outer radiation belt. Geophys Res Lett 30:1871. https://doi. org/10.1029/2003GL017698

Miyashita Y, Shinohara I, Fujimoto M, Hasegawa H, Hosokawa K, Takada T, Hori $\mathrm{T}$ (2011) A powerful tool for browsing quick-look data in solar-terrestrial physics: "Conjunction Event Finder". Earth Planets Space 63:e1-e4. https://doi.org/10.5047/eps2011.01.003

Miyoshi Y, Morioka A, Obara T, Misawa H, Nagai T, Kasahara Y (2003) Rebuilding process of the outer radiation belt during the 3 November 1993 magnetic storm: NOAA and Exos-D observations. J Geophys Res 108(A1):1004. https://doi.org/10.1029/2001JA007542

Miyoshi YS, Jordanova VK, Morioka A, Evans DS (2004) Solar cycle variations of the electron radiation belts: observations and radial diffusion simulation. Space Weather 2:S10S02. https://doi.org/10.1029/2004SW000070

Miyoshi Y, Morioka A, Kataoka R, Kasahara Y, Mukai T (2007) Evolution of the outer radiation belt during the November 1993 storms driven by corotating interaction regions. J Geophys Res 112:A05210. https://doi. org/10.1029/2006JA012148

Miyoshi Y, Kataoka R, Kasahara Y, Kumamoto A, Nagai T, Thomsen MF (2013) High-speed solar wind with southward interplanetary magnetic field causes relativistic electron flux enhancement of the outer radiation belt via enhanced condition of whistler waves. Geophys Res Lett 40:4520-4525. https://doi.org/10.1002/grl.50916

Miyoshi Y, Oyama S, Saito S, Kurita S, Fujiwara H, Kataoka R, Ebihara Y, Kletzing C, Reeves G, Santolik O, Clilverd M, Rodger CJ, Turunen E, Tsuchiya F (2015) Energetic electron precipitation associated with pulsating aurora: EISCAT and Van Allen Probe observations. J Geophys Res Space Phys 120:2754-2766. https://doi.org/10.1002/2014JA020690

Miyoshi Y, Kasaba Y, Shinohara I, Takashima T, Asamura K, Matsumoto H, Higashio N, Mitani T, Kasahara S, Yokota S, Wang S, Kazama Y, Kasahara Y, Yagitani S, Matsuoka A, Kojima H, Katoh Y, Shiokawa K, Seki K, Fujimoto M, Ono T, ERG Project Group (2017) Geospace exploration project: Arase (ERG). IOP Conf Ser J Phys Conf Ser 869:012095. https://doi. org/10.1088/1742-6596/869/1/012095

Nakamizo A, Hiraki Y, Ebihara Y, Kikuchi T, Seki K, Hori T, leda A, Miyoshi Y, Tsuji Y, Nishimura Y, Shinbori A (2012) Effect of R2-FAC development on the ionospheric electric field pattern deduced by a global ionospheric potential solver. J Geophys Res 117:A09231. https://doi. org/10.1029/2012JA017669

Nakamura S, Omura Y, Machida S, Shoji M, Nose M, Angelopoulos V (2014) Electromagnetic ion cyclotron rising tone emissions observed by THEMIS probes outside the plasmapause. J Geophys Res Space Phys 119:1874-1886. https://doi.org/10.1002/2013JA019146

Nakamura S, Omura Y, Shoji M, Nose M, Summers D, Angelopoulos V (2015) Subpacket structures in EMIC rising tone emissions observed by the THEMIS probes. J Geophys Res Space Phys 120:7318-7330. https://doi. org/10.1002/2014JA020764

Nakano S, Ueno G, Ebihara Y, Fok M-C, Ohtani S, Brandt PC, Mitchell DG, Keika $\mathrm{K}$, Higuchi T (2008) A method for estimating the ring current structure and the electric potential distribution using ENA data assimilation. J Geophys Res 113:A05208. https://doi.org/10.1029/2006JA011853

Nakano S, Fok M-C, Brandt PC, Higuchi T (2014) Estimation of temporal evolution of the helium plasmasphere based on a sequence of IMAGE/EUV images. J Geophys Res 119:3708-3723. https://doi. org/10.1002/2013JA019734

Nopper RW Jr, Carovillano RL (1978) Polar-equatorial coupling during magnetically active periods. Geophys Res Lett 5(8):699-702. https://doi. org/10.1029/GL005i008p00699

O'Brien TP, Moldwin MB (2003) Empirical plasmapause models from magnetic indices. Geophys Res Lett 30(4):1152. https://doi. org/10.1029/2002GL016007

Ober DM, Horwitz JL, Gallagher DL (1997) Formation of density troughs embedded in the outer plasmasphere by subauroral ion drift events. J Geophys Res 102:14595. https://doi.org/10.1029/97JA01046

Omura Y, Nunn D (2011) Triggering process of whistler mode chorus emissions in the magnetosphere. J Geophys Res 116:A05205. https://doi. org/10.1029/2010JA016280

Omura Y, Furuya N, Summers D (2007) Relativistic turning acceleration of resonant electrons by coherent whistler mode waves in a dipole magnetic field. J Geophys Res 112:A06236. https://doi.org/10.1029/2006JA012243

Omura Y, Katoh Y, Summers D (2008) Theory and simulation of the generation of whistler-mode chorus. J Geophys Res Space Phys 113:A04223. https://doi.org/10.1029/2007JA012622

Omura Y, Hikishima M, Katoh Y, Summers D, Yagitani S (2009) Nonlinear mechanisms of lower-band and upper-band VLF chorus emissions 
in the magnetosphere. J Geophys Res 114:A07217. https://doi. org/10.1029/2009JA014206

Omura Y, Pickett JS, Grison B, Santolik O, Dandouras I, Engebretson MJ, Decreau PME, Masson A (2010) Theory and observation of electromagnetic ion cyclotron chorus emissions in the magnetosphere. J Geophys Res 115:A07234. https://doi.org/10.1029/2010JA015300

Pembroke A, Toffoletto F, Sazykin S, Wiltberger M, Lyon J, Merkin V, Schmitt P (2012) Initial results from a dynamic coupled magnetosphere-ionosphere-ring current model. J Geophys Res Space Phys 117(2):A02211. https://doi.org/10.1029/2011JA016979

Pickett JS, Grison B, Omura Y, Engebretson MJ, Dandouras I, Masson A, Adrian ML, Santolik O, Decreau PME, Cornilleau-Wehrlin N, Constantinescu D (2010) Cluster observations of EMIC triggered emissions in association with PC1 waves near Earth's plasmapause. Geophys Res Lett 37:L09104

Picone JM, Hedin AE, Drob DP, Aikin AC (2002) NRLMSISE-00 empirical model of the atmosphere: statistical comparisons and scientific issues. J Geophys Res 107(A12):1468. https://doi.org/10.1029/2002JA009430

Ponomarenko PV, Waters CL, Sciffer MD et al (2001) Spatial structure of ULF waves: comparison of magnetometer and Super Dual Auroral Radar Network data. J Geophys Res 106:10509. https://doi. org/10.1029/2000JA000281

Reeves GD, McAdams KL, Friedel RHW, O'Brien TP (2003) Acceleration and loss of relativistic electrons during geomagnetic storms. Geophys Res Lett 30(10):1529. https://doi.org/10.1029/2002GL016513

Reeves GD et al (2013) Electron acceleration in the heart of the Van Allen radiation belts. Science 341:991-994. https://doi.org/10.1126/ science. 1237743

Roederer JG (1970) Dynamics of geomagnetically trapped radiation. Springer, Berlin

Ruohoniemi JM, Baker KB (1998) Large-scale imaging of high-latitude convection with Super Dual Auroral Radar Network HF radar observations. J Geophys Res Space Phys 103:20797-20811. https://doi. org/10.1029/98JA01288

Ruohoniemi JM, Greenwald RA (1998) The response of high-latitude convection to a sudden southward IMF turning. Geophys Res Lett 25:2913-2916

Saito S, Miyoshi Y, Seki K (2010) A split in the outer radiation belt by magnetopause shadowing: test particle simulations. J Geophys Res 115:A08210. https://doi.org/10.1029/2009JA014738

Saito S, Miyoshi Y, Seki K (2012) Relativistic electron microbursts associated with whistler chorus rising tone elements: GEMSIS-RBW simulations. J Geophys Res 117:A10206. https://doi.org/10.1029/2012JA018020

Saito S, Miyoshi Y, Seki K (2016) Rapid increase in relativistic electron flux controlled by nonlinear phase trapping of whistler chorus elements. J Geophys Res Space Phys 121:6573-6589. https://doi. org/10.1002/2016JA022696

Scherliess L, Fejer BG (1999) Radar and satellite global equatorial F region vertical drift model. J Geophys Res 104:6829-6842

Schulz M, Lanzerotti $\sqcup$ (1974) Particle diffusion in the radiation belts. Springer, New York

Schunk RW, Nagy AF (1978) Electron temperatures in the F region of the ionosphere: theory and observations. Rev Geophys 16:355-399. https:// doi.org/10.1029/RG016i003p00355

Schunk RW, Walker JCG (1973) Theoretical ion densities in the lower ionosphere. Planet Space Sci 21:1875-1896. https://doi. org/10.1016/0032-0633(73)90118-9

Seki K, Miyoshi Y, Summers D, Meredith NP (2005) Comparative study of outerzone relativistic electrons observed by Akebono and CRRES. J Geophys Res 110:A02203. https://doi.org/10.1029/2004JA010655

Senior C, Blanc M (1984) On the control of magnetospheric convection by the spatial distribution of ionospheric conductivities. J Geophys Res 89(A1):261-284. https://doi.org/10.1029/JA089iA01 p00261

Shiokawa K et al (2017) Ground-based instruments of the PWING project to investigate dynamics of the inner magnetosphere at subauroral latitudes as a part of the ERG-ground coordinated observation network. Earth Planets Space 69:160. https://doi.org/10.1 186/s40623-017-0745-9

Shoji M, Omura Y (2011) Simulation of electromagnetic ion cyclotron triggered emissions in the Earth's inner magnetosphere. J Geophys Res 116:A05212. https://doi.org/10.1029/2010JA016351

Shoji M, Omura Y (2012) Precipitation of highly energetic protons by helium branch electromagnetic ion cyclotron triggered emissions. J Geophys Res 117:A12210. https://doi.org/10.1029/2012JA017933
Shoji M, Omura Y (2013) Triggering process of electromagnetic ion cyclotron rising tone emissions in the inner magnetosphere. J Geophys Res 118:5553-5561. https://doi.org/10.1002/jgra.50523

Shoji M, Omura Y (2014) Spectrum characteristics of electromagnetic ion cyclotron triggered emissions and associated energetic proton dynamics. J Geophys Res 119:3480-3489. https://doi. org/10.1002/2013JA019695

Shprits YY, Thorne RM, Friedel R, Reeves GD, Fennell J, Baker DN, Kanekal SG (2006) Outward radial diffusion driven by losses at magnetopause. J Geophys Res 111:A11214. https://doi.org/10.1029/2006JA011657

Shprits YY, Elkington SR, Meredith NP, Subbotin DA (2008a) Review of modeling of losses and sources of relativistic electrons in the outer radiation belt l: radial transport. J Atmos Sol Terr Phys 70:1679-1693. https://doi. org/10.1016/j.jastp.2008.06.008

Shprits YY, Elkington SR, Meredith NP, Subbotin DA (2008b) Review of modeling of losses and sources of relativistic electrons in the outer radiation belt II: local acceleration and loss. J Atmos Sol Terr Phys 70:1694-1713. https://doi.org/10.1016/j.jastp.2008.06.014

Shprits YY, Subbotin D, Ni B (2009) Evolution of electron fluxes in the outer radiation belt computed with the VERB code. J Geophys Res 114:A11209. https://doi.org/10.1029/2008JA013784

Shprits YY, Subbotin D, Drozdov A, Usanova ME, Kellerman A, Orlova K, Baker DN, Turner DL, Kim KC (2013) Unusual stable trapping of the ultrarelativistic electrons in the Van Allen radiation belts. Nat Phys 9:699-703. https://doi.org/10.1038/nphys2760

Sibeck DG, McEntire RW, Lui ATY, Lopez RE, Krimigis SM (1987) Magnetic field drift shell splitting: cause of unusual dayside particle pitch angle distributions during storms and substorms. J Geophys Res 92(A12):1348513497. https://doi.org/10.1029/JA092iA12p13485

Stern DP (1975) The motion of a proton in the equatorial magnetosphere. J Geophys Res. https://doi.org/10.1029/JA080i004p00595

Subbotin DA, Shprits YY (2009) Three-dimensional modeling of the radiation belts using the Versatile Electron Radiation Belt (VERB) code. Space Weather 7:S10001. https://doi.org/10.1029/2008SW000452

Sugiyama H, Singh S, Omura Y, Shoji M, Nunn D, Summers D (2015) Electromagnetic ion cyclotron waves in the Earth's magnetosphere with a kappa-Maxwellian particle distribution. J Geophys Res Space Phys 120:8426-8439. https://doi.org/10.1002/2015JA021346

Summers D, Thorne RM, Xiao F (1998) Relativistic theory of wave-particle resonant diffusion with application to electron acceleration in the magnetosphere. J Geophys Res 103(A9):20487-20500

Tanaka T (1995) Generation mechanisms for magnetosphere-ionosphere current systems deduced from a three-dimensional MHD simulation of the solar wind-magnetosphere-ionosphere coupling processes. J Geophys Res 100(A7):12057-12074. https://doi.org/10.1029/95JA00419

Tanaka T (2015) Substorm auroral dynamics reproduced by advanced global magnetosphere-ionosphere $(\mathrm{M}-\mathrm{I})$ coupling simulation. Auroral dynamics and space weather. Wiley, Hoboken, pp 177-190

Tanaka T, Ebihara Y, Watanabe M, Den M, Fujita S, Kikuchi T, Hashimoto KK, Kataoka R (2017) Global simulation study for the time sequence of events leading to the substorm onset. J Geophys Res Space Phys 122:6210-6239. https://doi.org/10.1002/2017JA024102

Tsunomura S (1999) Numerical analysis of global ionospheric current system including the effect of equatorial enhancement. Ann Geophys 17:692-706. https://doi.org/10.1007/s00585-999-0692-2

Tsunomura S, Araki T (1984) Numerical analysis of equatorial enhancement of geomagnetic sudden commencement. Planet Space Sci 32:599-604. https://doi.org/10.1016/0032-0633(84)90109-0

Tsyganenko NA, Sitnov MI (2005) Modeling the dynamics of the inner magnetosphere during strong geomagnetic storms. J Geophys Res 110:A03208. https://doi.org/10.1029/2004JA010798

Tu J-N, Horwitz JL, Song P, Huang XQ, Reinisch BW, Richards PG (2003) Simulating plasmaspheric field-aligned density profiles measured with IMAGE/ RPI: Effects of plasmasphere refilling and ion heating. J Geophys Res 108(A1):1017. https://doi.org/10.1029/2002JA009468

Ukhorskiy AY, Sitnov MI, Takahashi K, Anderson BJ (2009) Radial transport of radiation belt electrons due to stormtime Pc5 waves. Ann Geophys 27:2173-2181

Umemura N, Segawa T, Miyashita Y, Keika K, Miyoshi Y, Hori T, Shoji M, Tanaka Y, Seki K, Shinohara I (2016) Development and future plans of the web based data analysis tool ERGWAT. J Space Sci Info Jpn 
4:25-31, JAXA-RR-16-007 (ISSN 1349-1113). https://doi.org/10.20637/ jaxa-rr-16-007/0003

Volland H (1973) A semiempirical model of large-scale magnetospheric electric fields. J Geophys Res 78:171. https://doi.org/10.1029/ JA078i001p00171

Watanabe S, Whalen BA, Yau AW (1992) Thermal ion observations of depletion and refilling in the plasmaspheric trough. J Geophys Res 97:1081-1096

Webb PA, Essex EA (2001) A dynamic diffusive equilibrium model of the ion densities along plasmaspheric magnetic flux tubes. J Atmos Solar Terr Phys 63:1249-1260

Weimer DR (2000) A new technique for the mapping of ionospheric fieldaligned currents from satellite magnetometer data. In: Ohtani $S$ et al (ed) Magnetospheric current systems. Geophys Monogr Ser, 118:381. AGU, Washington, DC
Weimer DR (2001) An improved model of ionospheric electric potentials including substorm perturbations and application to the Geospace Environment Modeling November 24, 1996, event. J Geophys Res 106:407-416. https://doi.org/10.1029/2000JA000604

Williams DJ (1987) Ring current and radiation belts. Rev Geophys 25(3):570578. https://doi.org/10.1029/RG025i003p00570

Xiao F, Su Z, Zheng H, Wang S (2010) Three-dimensional simulations of outer radiation belt electron dynamics including cross-diffusion terms. J Geophys Res 115:A05216. https://doi.org/10.1029/2009JA014541 